\title{
Homological realization of Nakajima varieties and Weyl group actions
}

\author{
Igor Frenkel, Mikhail Khovanov and Olivier Schiffmann
}

\begin{abstract}
We give a realization of Nakajima varieties and the action of the Weyl group on them using certain canonical structures of homological algebras and their natural generalization, which we develop in this paper. We consider in detail the case of an affine quiver, where we present a simple homological characterization of Nakajima varieties and its relation to moduli of sheaves on the projective plane.
\end{abstract}

\section{Contents}

1 Nakajima varieties

1482

2 Categories of $(A, c)$-complexes

3 Categories of duplexes

4 Stable categories

1488

5 Homological realization of Nakajima varieties

1488

6 Weyl group action in categories of duplexes

1491

7 Weyl group actions on Nakajima varieties

8 Nakajima varieties for affine quivers

9 Koszul duality and sheaves on $\mathbb{P}^{2}$

1499

References

1502

\section{Introduction}

A geometric approach to the representation theory of Kac-Moody algebras was given by Nakajima in the groundbreaking work [Nak94], which was a culmination of a series of remarkable discoveries discussed in the introduction to his paper. For any simply laced Kac-Moody algebra $\mathfrak{g}$ with triples of generators $\left(e_{a}, h_{a}, f_{a}\right)_{a \in I}$ indexed by a finite set $I$, Nakajima constructed a family of complex varieties $\mathcal{M}_{\zeta}(\mathbf{v}, \mathbf{w})$, where $\mathbf{v}, \mathbf{w} \in \mathbb{N}^{I}$ and $\zeta \in \mathbb{R}^{3} \otimes \mathbb{R}^{I}$ such that, for any generic $\zeta$,

$$
\operatorname{dim} H^{\mathrm{mid}}\left(\mathcal{M}_{\zeta}(\mathbf{v}, \mathbf{w})\right)=\operatorname{dim} L_{\lambda}[\lambda-\alpha],
$$

where $L_{\lambda}$ is the integrable highest weight $\mathfrak{g}$-module of highest weight $\lambda$, where $L_{\lambda}[\lambda-\alpha]$ is the corresponding weight space, and where

$$
\alpha=\sum_{a} v_{a} \alpha_{a}, \quad \lambda=\sum_{a} w_{a} \omega_{a}
$$

for $\left(\alpha_{a}\right)_{a \in I},\left(\omega_{a}\right)_{a \in I}$ the set of simple roots and fundamental weights, respectively. Nakajima realized the action of the generators $\left(e_{a}, h_{a}, f_{a}\right)_{a \in I}$ in a geometric way, the contravariant form on $L_{\lambda}$, and

Received 10 March 2004, accepted in final form 25 February 2005. 2000 Mathematics Subject Classification 17B20, 16G20, 14D20.

Keywords: Nakajima quivers varieties, Weyl group, Koszul duality.

This journal is (C) Foundation Compositio Mathematica 2005. 


\section{Frenkel, M. Khovanov and O. Schiffmann}

indicated the geometric meaning of the Weyl group action on weight spaces

$$
\sigma: L_{\lambda}[\lambda-\alpha] \stackrel{\sim}{\rightarrow} L_{\lambda}[\sigma(\lambda-\alpha)]
$$

The action of the Weyl group on the underlying quiver varieties was further developed by Lusztig [Lus00], Maffei [Maf02], and Nakajima [Nak03].

Nakajima varieties are defined in terms of certain data attached to the Dynkin diagram $\mathbf{Q}$ of the Kac-Moody algebra $\mathfrak{g}$. This data can be viewed as a generalization of the Atiyah-DrinfeldHitchin-Manin description of the instanton moduli spaces. Correspondingly, various structures of representation theory of Kac-Moody algebra $\mathfrak{g}$ including the action of the Weyl group on highest weight modules were described in terms of this linear data. We will review the original constructions relevant to our present work in $\S 1$.

In this paper, we interpret the data and, consequently, Nakajima varieties, via differential graded modules over a finite-dimensional quotient $A(\mathbf{Q})$ of the double path algebra of $\mathbf{Q}$. Defining relations in this quotient algebra depend on the choice of orientation $\epsilon$ of $\mathbf{Q}$, but different orientations produce isomorphic algebras. The algebra $A(\mathbf{Q})$ is the quadratic dual of the preprojective algebra of the (oriented) graph $\mathbf{Q}$ and has a Frobenius structure. If $\mathbf{Q}$ is bipartite (see $\S 5$ ), $A(\mathbf{Q})$ is isomorphic to the zigzag algebra of $\mathbf{Q}$ studied in [HK01]. Let us denote $A(\mathbf{Q})$ simply by $A$.

Our realization of the Nakajima varieties allows us to view their theory in the context of homological algebra. In particular, simple $A$-modules $S_{a}$ and projective $A$-modules $P_{a}$ are the basic building blocks in our picture. Furthermore, we give a natural interpretation of the Weyl group action on Nakajima varieties, with the simple reflection $s_{a}$ acting as homological 'addition' and 'substraction' of the projective module $P_{a}$. This Weyl group action comes from a modification of the braid group action in the derived category of $A$-modules, see [KS02, ST01, RZ03, HK01].

The theory of Nakajima varieties also suggest certain generalizations of some classical notions and results in homological algebra. In fact, our constructions depend in an essential way on the value of the parameter $\zeta=\left(\zeta_{\mathbb{R}}, \zeta_{\mathbb{C}}\right)$, where $\zeta_{\mathbb{R}} \in \mathbb{R}^{I}$ and $\zeta_{\mathbb{C}} \in \mathbb{C}^{I}$. If $\zeta_{\mathbb{C}}=0$ then we use the standard theory of differential graded algebras and complexes of modules over them. On the other hand, when $\zeta_{\mathbb{C}} \neq 0$ we are led to consider modules over $A$ equipped with a generalized differential $d$, which is a degree 1 map satisfying

$$
d^{2}=c
$$

for a suitable central element $c \in A$. We introduce categories of $(A, c)$-complexes in $\S 2$.

To define the Weyl group action we are then forced into 2-periodic generalized complexes, which we call duplexes. The theory of duplexes, which we outline in $\S 3$, can be developed in parallel with some classical results of homological algebra and seems to be worthy of a deeper, independent study.

In $\S 4$ we consider another way of deforming the derived category of $A$-modules: by a theorem of Happel [Hap88], this derived category is equivalent to the stable category of graded modules over the algebra $A \dot{\otimes} \mathbb{C}[d] / d^{2}$, where $\dot{\otimes}$ denotes the super tensor product. We introduce the stable category of $A \dot{\otimes} \mathbb{C}[d] /\left\langle d^{2}-c\right\rangle$ and relate this to the category of $(A, c)$-duplexes.

Our interpretation of Nakajima varieties $\mathcal{M}_{\zeta}(\mathbf{v}, \mathbf{w})$ is given in $\S 5$, and is set-theoretic, consisting of a bijection between the set of points of $\mathcal{M}_{\zeta}(\mathbf{v}, \mathbf{w})$ and certain isomorphism classes of differential $A$-modules. Let $\left(V_{a}\right)_{a \in I},\left(W_{a}\right)_{a \in I}$ denote collections of vector spaces of dimensions $\left(v_{a}\right)_{a \in I}$ and $\left(w_{a}\right)_{a \in I}$, respectively. We consider $\mathbb{Z} / 2 \mathbb{Z}$-graded $A$-modules $M$ equipped with a generalized differential $d$ such that $d^{2}=c$, where $c$ is $\zeta_{\mathbb{C}}$, viewed as a central element of $A$, and

$$
M \cong \bigoplus_{a}\left(P_{a} \otimes V_{a} \oplus S_{a}[-1] \otimes W_{a}\right),
$$

where $[-1]$ denotes a grading shift, and the isomorphism is that of $A$-modules. When $\zeta_{\mathbb{C}}$ is not generic (for example, $\zeta_{\mathbb{C}}=0$ ), we add an irreducibility condition with respect to $d$, analogous 


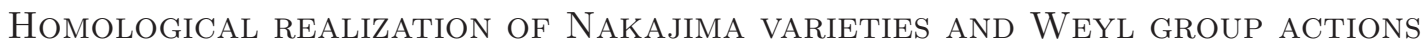

to a stability condition. We show that the set of classes of pairs $(M, d)$ as above is in a natural bijection with the set of $G_{W}$-orbits of the quiver variety $\mathcal{M}_{\zeta}(\mathbf{v}, \mathbf{w})$, where $G_{W}=\prod_{a} G L\left(W_{a}\right)$. The full variety $\mathcal{M}_{\zeta}(\mathbf{v}, \mathbf{w})$ is obtained by fixing, in addition, an isomorphism (framing) $W_{a} \simeq$ $\operatorname{Hom}_{A}\left(S_{a}[-1], M\right)$ for $a \in I$.

To obtain a realization of the Weyl group action, we consider a duplex of $A$-bimodules $C_{a, x}$ associated to a vertex $a$ of $Q$ :

$$
\rightarrow P_{a} \otimes{ }_{a} P \rightarrow A \rightarrow
$$

where $x \in \mathbb{C}$ and one of the bimodule maps depends on $x$. We show in $\S 6$ that duplexes $C_{a, x}$ are invertible in the homotopy category (as well as in the stable category),

$$
C_{a, x} \otimes_{A} C_{a,-x} \cong A,
$$

and satisfy Yang-Baxter relations:

$$
C_{a, x} \otimes C_{b, y} \cong C_{b, y} \otimes C_{a, x}
$$

for any two vertices $a$ and $b$ which are not joined by an edge, and

$$
C_{a, x} \otimes C_{b, x+y} \otimes C_{a, y} \cong C_{b, y} \otimes C_{a, x+y} \otimes C_{b, x}
$$

for $a$ and $b$ joined by a single edge. In the limit $x \rightarrow 0$ our duplexes degenerate into those used to categorify the Burau representation of the braid group, see [KS02].

Points of Nakajima varieties $\mathcal{M}_{\zeta}(\mathbf{v}, \mathbf{w})$ can be identified with certain isomorphism classes of $A$-duplexes. The functor of the tensor product with the bimodule duplex $C_{a, x}$ acts in categories of $A$-duplexes and restricts to a bijection

$$
\mathcal{R}_{a}: \mathcal{M}_{\zeta}(\mathbf{v}, \mathbf{w}) \stackrel{\sim}{\rightarrow} \mathcal{M}_{s_{a} \cdot \zeta}\left(s_{a} \cdot(\mathbf{v}, \mathbf{w})\right),
$$

where $s_{a}$ is a simple reflection. We show in $\S 7$ that our reflection maps coincide with those in [Lus00, Maf02, Nak03].

The class of Kac-Moody algebras associated to affine Dynkin diagrams plays a very special role in representation theory. The corresponding class of quivers is also distinguished in the Nakajima theory since it appears in the study of the instanton moduli spaces. It is well known that simply-laced affine Dynkin diagrams are in a bijection with finite subgroups $\Gamma \subset S L(2, \mathbb{C})$ and it is natural to reformulate Nakajima's work entirely in terms of these finite groups. In $\S 8$ we recast our realization of Nakajima varieties in this light. We replace $A$ by the Morita equivalent algebra $A_{\Gamma}=\Lambda \rho \otimes \mathbb{C}[\Gamma]$, where $\rho$ is the natural two-dimensional representation of $\Gamma$. To a collection of vector spaces $\left(V_{a}\right)_{a \in I}$ and $\left(W_{a}\right)_{a \in I}$ we now associate two $\Gamma$-modules

$$
\mathbb{V}=\bigoplus_{a} \rho_{a} \otimes V_{a}, \quad \mathbb{W}=\bigoplus_{a} \rho_{a} \otimes W_{a},
$$

where $\left(\rho_{a}\right)_{a \in I}$ is the set of all irreducible representations of $\Gamma$. The module $M$ in $(0.1)$ is replaced by the following

$$
M=\Lambda \rho \otimes \mathbb{V} \oplus \mathbb{W}[-1] .
$$

The latter can be viewed as a module over $\widetilde{A}_{\Gamma, c}=A_{\Gamma} \dot{\otimes} \mathbb{C}[d] /\left\langle d^{2}-c\right\rangle$, where $c$ is a central element of $A_{\Gamma}$ which depends linearly on $\zeta_{\mathbb{C}}$. It turns out that we can characterize modules of the form $(0.2)$ as a certain class of elements of the stable category of $\mathbb{Z} / 2 \mathbb{Z}$-graded $\widetilde{A}_{\Gamma, c}$-modules $\underline{\operatorname{Mod}}_{2}\left(\widetilde{A}_{\Gamma, c}\right)$. This yields a realization of Nakajima varieties via isomorphism classes of pairs $(M, u)$, where $M$ is an object of $\underline{\operatorname{Mod}}_{2}\left(\widetilde{A}_{\Gamma, c}\right)$ and $u: \mathbb{W} \rightarrow R(M)$ is a fixed isomorphism, with $R(M)$ being the restriction of the module $M$ to $A_{\Gamma}$.

The relation between this realization of the Nakajima varieties by means of the stable category $\underline{\operatorname{Mod}}_{2}\left(\widetilde{A}_{\Gamma, c}\right)$ and the realization as a moduli space of $\Gamma$-equivariant torsion-free sheaves on a noncommutative $\mathbb{P}^{2}$ with fixed framing at infinity (presented in $[\mathrm{BGK}]$ ) is explained in $\S 9$. In fact, one 


\section{Frenkel, M. Khovanov and O. Schiffmann}

may view such torsion-free sheaves as modules over the algebra Koszul dual to $\widetilde{A}_{\Gamma, c}$, see [BGK, Appendix B]. Thus, our construction illustrates a noncommutative version of the classical theorem of [BGG78] claiming that when $\Gamma=\{e\}$ and $c=0$ (the case of commutative $\mathbb{P}^{2}$ ), the derived category of coherent sheaves over $\mathbb{P}^{2}$ is equivalent to the stable category of $\widetilde{A}_{\Gamma, c}$. Finally, we reformulate the action of the Weyl group on quiver varieties via certain natural duplexes of $A_{\Gamma}$-bimodules.

We believe that the realization of the Nakajima varieties and Weyl group actions by means of natural categorical constructions presented in this paper is only a first step in a more general program of recasting the Nakajima geometric approach to representation theory of Kac-Moody algebras in terms of canonical structures of homological algebra. We hope that the emerging interaction between the two areas will be beneficial to both subjects. Below we will make a few remarks about further developments of both areas inspired by our constructions.

As we mentioned in the beginning of the introduction, the Nakajima varieties encode the structure of the integrable highest weight modules $L_{\lambda}$. The latter modules possess rich structures associated with the corresponding Weyl group W. On the one hand, each module $L_{\lambda}$ contains a family of Demazure submodules $L_{\lambda, w}$ defined for any $w \in \mathbf{W}$. On the other hand, each module $L_{\lambda}$ admits the Bernštel̆n-Gel'fand-Gel'fand (BGG) resolution by Verma modules $V_{w \cdot \lambda}$, where again $w \in \mathbf{W}$ (see, e.g., [Kum02] for a review of both constructions). It is natural to expect that our realization of the action of $\mathbf{W}$ on Nakajima varieties should lead to a transparent geometric construction of the Demazure modules as well as the BGG resolution.

Concerning the applications to homological algebra, the example studied in this paper already suggests the following generalizations of algebraic structures:

$$
\begin{gathered}
\text { abelian } \longrightarrow \text { triangulated } \\
\mathbb{Z} \text {-graded } \longrightarrow \mathbb{Z} / 2 \mathbb{Z} \text {-graded } \\
d^{2}=0 \longrightarrow d^{2}=c .
\end{gathered}
$$

Starting with an abelian category of modules over a ring, we pass to the triangulated category of complexes (the top arrow in the diagram). This arrow is the familiar advancement from the classical theory of modules over a ring to homological algebra. The bottom arrows refer to two more recent developments where:

- one gains from working with periodic triangulated categories, those with $[2 k] \cong$ Id for some $k$ (case $k=1$ seems especially important);

- differential modules acquire curvature (the square of differential is no longer zero).

Both transformations are natural from the deformation theory viewpoint. When the cohomology ring of a symplectic manifold is deformed to the quantum cohomology ring, its $\mathbb{Z}$-grading collapses to a $\mathbb{Z} / 2 k \mathbb{Z}$-grading, where $k$ is the minimal Chern number (see [MS94, Section 1.7]), and the shift functor in the $A_{\infty}$ triangulated Fukaya-Floer category of the manifold is periodic, $[2 k] \cong \mathrm{Id}$. Deforming $d^{2}=0$ to $d^{2}=c$ is as legitimate as deforming the ring structure, when one is describing all deformations of the homotopy category of modules over a (graded) ring. We should also mention the paper of Peng and Xiao [PX00], where 2-periodic triangulated categories appear in relation to Hall algebras.

\section{Nakajima varieties}

We recall the definition of Nakajima quiver varieties. Let $\mathbf{Q}=(I, E)$ be an arbitrary finite graph with $I$ the set of vertices and $E$ the set of edges. We allow $\mathbf{Q}$ to have loops and multiple edges. Let $H$ be the set of oriented edges of this graph (thus $H$ is 'twice as large' as $E$ ). For any $h \in H$ we 
Homological Realization of NAKajima varieties AND Weyl group aCtions
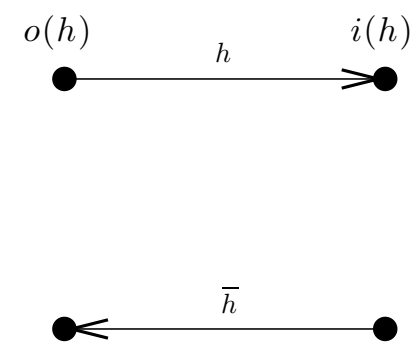

Figure 1. Two orientations of an edge.

denote by $o(h)$ and $i(h)$ the outgoing and incoming vertices of $h$, respectively, and by $\bar{h}$ the edge $h$ with the opposite orientation, see Figure 1.

Let $(\mathbf{v}, \mathbf{w}) \in \mathbb{N}^{I} \times \mathbb{N}^{I}$ with $\mathbf{v}=\left(v_{a}\right)_{a \in I}$ and $\mathbf{w}=\left(w_{a}\right)_{a \in I}$. Fix some $I$-graded $\mathbb{C}$-vector spaces $V=\bigoplus V_{a}$ and $W=\bigoplus W_{a}$ such that $\operatorname{dim} V=\mathbf{v}$ and $\operatorname{dim} W=\mathbf{w}$. Set

$$
\begin{gathered}
E(V, V)=\bigoplus_{h \in H} \operatorname{Hom}\left(V_{o(h)}, V_{i(h)}\right), \\
L(V, W)=\bigoplus_{a} \operatorname{Hom}\left(V_{a}, W_{a}\right), \quad L(W, V)=\bigoplus_{a} \operatorname{Hom}\left(W_{a}, V_{a}\right)
\end{gathered}
$$

and

$$
\mathbf{M}(\mathbf{v}, \mathbf{w})=E(V, V) \oplus L(W, V) \oplus L(V, W) .
$$

An element of $\mathbf{M}(\mathbf{v}, \mathbf{w})$ will usually be denoted by its components $(B, i, j)$.

Let $\epsilon: H \rightarrow\{1,-1\}$ be any function satisfying $\epsilon(h)+\epsilon(\bar{h})=0$ for all $h \in H$. Such functions are in a bijection with orientations of $\mathbf{Q}$, the $\epsilon$-orientation consists of all edges $h$ with $\epsilon(h)=1$. Consider the maps

$$
\begin{aligned}
\mu_{\mathbb{C}}: \mathbf{M}(\mathbf{v}, \mathbf{w}) & \longrightarrow \bigoplus_{a} \mathfrak{g l}\left(V_{a}\right), \\
(B, i, j) & \longrightarrow\left(\sum_{o(h)=a} \epsilon(h) B_{\bar{h}} B_{h}+i_{a} j_{a}\right)_{a},
\end{aligned}
$$

and

$$
\begin{aligned}
\mu_{\mathbb{R}}: \mathbf{M}(\mathbf{v}, \mathbf{w}) & \longrightarrow \bigoplus_{a} \mathfrak{u}\left(V_{a}\right), \\
(B, i, j) & \mapsto \frac{\sqrt{-1}}{2}\left(\sum_{o(h)=a} B_{\bar{h}} B_{\bar{h}}^{\star}-B_{h}^{\star} B_{h}+i_{a} i_{a}^{\star}-j_{a}^{\star} j_{a}\right)_{a} .
\end{aligned}
$$

In the above, $f^{\star}$ denotes the Hermitian adjoint of $f$. Following Nakajima, to $\zeta_{\mathbb{R}} \in \mathbb{R}^{I}$ we associate a central element $\zeta_{\mathbb{R}}=\sum_{a}\left(\sqrt{-1} \zeta_{\mathbb{R}, a} / 2\right) \operatorname{Id} \in \bigoplus_{a} \mathfrak{u}\left(V_{a}\right)$, and to $\zeta_{\mathbb{C}} \in \mathbb{C}^{I}$ we associate a central element $\zeta_{\mathbb{C}}=\bigoplus_{a} \zeta_{\mathbb{C}, a} \operatorname{Id} \in \bigoplus_{a} \mathfrak{g l}\left(V_{a}\right)$. The group $U_{V}=\prod_{a} U\left(V_{a}\right)$ acts on $\mathbf{M}(\mathbf{v}, \mathbf{w})$ by conjugation. Finally, we put

$$
\mathcal{M}_{\zeta}(\mathbf{v}, \mathbf{w})=\left(\mu_{\mathbb{R}} \times \mu_{\mathbb{C}}\right)^{-1}\left(\boldsymbol{\zeta}_{\mathbb{R}}, \boldsymbol{\zeta}_{\mathbb{C}}\right) / U_{V}
$$

where $\zeta=\left(\zeta_{\mathbb{R}}, \zeta_{\mathbb{C}}\right)$. Different choices of $\epsilon$ yield isomorphic varieties.

1.1 When $\zeta_{\mathbb{R}} \in \mathbb{Z}^{I}$ there is also a purely complex-geometric description of $\mathcal{M}_{\zeta}(\mathbf{v}, \mathbf{w})$. Note that the group $G_{V}=\prod_{a} G L\left(V_{a}\right)$ acts on $\mathbf{M}(\mathbf{v}, \mathbf{w})$ by conjugation. To $\zeta_{\mathbb{R}}$ we associate 


\section{Frenkel, M. Khovanov and O. Schiffmann}

the character $\chi_{\zeta_{\mathbb{R}}}: G_{V} \rightarrow \mathbb{C}^{*},\left(g_{a}\right)_{a} \mapsto \prod_{a} \operatorname{det}\left(g_{a}\right)^{\zeta_{\mathbb{R}, a}}$. Following Nakajima [Nak94], we have

$$
\mathcal{M}_{\zeta}(\mathbf{v}, \mathbf{w}) \simeq \operatorname{Proj}\left(\bigoplus_{n \geqslant 0} A_{\zeta_{\mathbb{R}}}^{n}(\mathbf{v}, \mathbf{w})\right),
$$

where

$$
A_{\zeta_{\mathbb{R}}}^{n}(\mathbf{v}, \mathbf{w})=\left\{f \in \mathbb{C}\left[\mu_{\mathbb{C}}^{-1}\left(\boldsymbol{\zeta}_{\mathbb{C}}\right)\right] \mid f(g \cdot(B, i, j))=\chi_{\zeta_{\mathbb{R}}}(g)^{n} f((B, i, j)) \forall g \in G_{V}\right\} .
$$

There is an open subset $\mathbf{M}_{\zeta}^{s s}(\mathbf{v}, \mathbf{w}) \subset \mu_{\mathbb{C}}^{-1}\left(\boldsymbol{\zeta}_{\mathbb{C}}\right)$ of semistable points such that $\mathcal{M}_{\zeta}(\mathbf{v}, \mathbf{w})=$ $\mathbf{M}_{\zeta}^{s s}(\mathbf{v}, \mathbf{w}) / / G_{V}$ (categorical quotient). We will mainly be interested in the following two cases.

(i) $\zeta_{\mathbb{R}} \in\left(\mathbb{N}^{+}\right)^{I}$ and $\zeta_{\mathbb{C}}=0$. In this case $(B, i, j) \in \mathbf{M}_{\zeta_{\mathbb{C}}}^{s s}(\mathbf{v}, \mathbf{w})$ if the following condition is satisfied: the only (graded) $B$-invariant subspace of $V$ contained in Ker $j$ is $\{0\}$.

(ii) $\zeta_{\mathbb{R}}$ is arbitrary and $\zeta_{\mathbb{C}}$ satisfies the following genericity condition: for every $n_{1}, \ldots, n_{k} \in \mathbb{Z}$, we have $\sum_{a} n_{a} \zeta \mathbb{C}, a=0 \Rightarrow n_{a}=0$ for all $a$. In this case, all points $(B, i, j)$ in $\mu_{\mathbb{C}}^{-1}\left(\boldsymbol{\zeta}_{\mathbb{C}}\right)$ are semistable (and, in fact, all points $(B, i, j)$ in $\mu_{\mathbb{C}}^{-1}\left(\boldsymbol{\zeta}_{\mathbb{C}}\right)$ automatically satisfy the condition in case (i)).

In the two above cases, $G_{V}$ acts freely on $\mathbf{M}_{\zeta}^{s s}(\mathbf{v}, \mathbf{w})$ (see, e.g., [Nak94]), so that the categorical quotients are actually geometric (smooth) quotients. Note also that, in case (ii), the variety $\mathcal{M}_{\zeta}(\mathbf{v}, \mathbf{w})$ is actually independent of $\zeta_{\mathbb{R}}$.

1.2 To the graph $(I, E)$ we associate a symmetric $|I| \times|I|$ Borcherds matrix $A=\left(a_{i j}\right)$ with

$$
a_{i j}=2 \delta_{i j}-\#\{h \in H \mid i(h)=i, o(h)=j\} .
$$

Let $I^{r e} \subset I$ be the set of all loopless vertices (characterized by the relation $a_{i i}=2$ ). To $A$ corresponds a Borcherds algebra (or generalized Kac-Moody algebra) $\mathfrak{g}$ (see [Bor88]). Let us fix a Cartan decomposition $\mathfrak{g}=\mathfrak{n}_{-} \oplus \mathfrak{h} \oplus \mathfrak{n}_{+}$and let $\alpha_{a}$ and $\omega_{a}$ (respectively $\alpha^{\vee}$ and $\omega_{a}^{\vee}$ ) stand for the simple root and fundamental weight (respectively simple coroot and fundamental coweight) associated to a vertex $a$. We put

$$
Q=\bigoplus_{s} \mathbb{Z} \alpha_{a}, \quad P=\bigoplus_{a} \mathbb{Z} \omega_{a}
$$

We also set $Q^{\vee}=\bigoplus_{a} \mathbb{Z} \alpha_{a}^{\vee}$ and $P^{\vee}=\bigoplus_{a} \mathbb{Z} \omega_{a}^{\vee}$ and we denote by $\langle\cdot, \cdot\rangle$ the natural pairing between $\mathfrak{h}$ and $\mathfrak{h}^{*}$. We consider $\mathbf{v}, \mathbf{w}, \zeta_{\mathbb{R}}$ and $\zeta_{\mathbb{C}}$ as elements of $\mathfrak{h}^{*}$ via the identifications

$$
\mathbf{v} \mapsto \sum_{a} v_{a} \alpha_{a}, \quad \mathbf{w} \mapsto \sum_{a} w_{a} \omega_{a}, \quad \zeta_{\mathbb{R}} \mapsto \sum_{a} \zeta_{\mathbb{R}, a} \omega_{a}, \quad \zeta_{\mathbb{C}} \mapsto \sum_{a} \zeta_{\mathbb{C}, a} \omega_{a}
$$

1.3 We say that the parameter $\zeta$ is generic when

$$
\text { For every } \nu \in P^{\vee} \text {, we have }\left\langle\nu, \zeta_{\mathbb{R}}\right\rangle \neq 0 \text { or }\left\langle\nu, \zeta_{\mathbb{C}}\right\rangle \neq 0
$$

The variety $\mathcal{M}_{\zeta}(\mathbf{v}, \mathbf{w})$ is smooth whenever $\zeta$ is generic. Moreover (for fixed $\mathbf{v}$ and $\mathbf{w}$ ), the varieties corresponding to generic parameters are all diffeomorphic [Nak94, Corollary 4.2].

1.4 The Weyl group W of $\mathfrak{g}$ is defined to be the subgroup of Aut $\left(\mathfrak{h}^{*}\right)$ generated by reflections

$$
s_{a}: \alpha \mapsto \alpha-\left\langle\alpha, \alpha_{a}^{\vee}\right\rangle \alpha_{a}
$$

for $a \in I^{r e}$. Note that $\mathbf{W}$ acts on $Q$. The dual action on $Q^{\vee}$ is given by

$$
s_{a}: \alpha^{\vee} \mapsto \alpha^{\vee}-\left\langle\alpha_{a}, \alpha^{\vee}\right\rangle \alpha_{a}^{\vee} .
$$

Moreover, $\langle\cdot, \cdot\rangle$ induces a perfect pairing between $P$ and $Q^{\vee}$, and thus (1.2) gives rise to an action of $\mathbf{W}$ on $P$ by duality. 


\section{Homological REALIZATION OF NAKAJIMA VARIETIES AND WEYL GROUP ACTIONS}

With this convention, $\mathbf{W}$ acts on $\zeta_{\mathbb{R}}$ and $\zeta_{\mathbb{C}}$ via the identifications in $\S 1.2$. We also define an action on pairs $(\mathbf{v}, \mathbf{w})$ by $\sigma \cdot(\mathbf{v}, \mathbf{w}):=(\sigma(\mathbf{v}-\mathbf{w})+\mathbf{w}, \mathbf{w})$. Following [Lus00] (see also [Nak94]), Maffei [Maf02] defined, for generic $\zeta$, isomorphisms

$$
\kappa_{\sigma}: \mathcal{M}_{\zeta}(\mathbf{v}, \mathbf{w}) \stackrel{\sim}{\rightarrow} \mathcal{M}_{\sigma \cdot \zeta}(\sigma \cdot(\mathbf{v}, \mathbf{w})) .
$$

Let us consider the situation when $\zeta_{\mathbb{C}}$ is generic as in $\S 1.1$ case (ii) (so that $\zeta$ is generic). In that situation, it is more convenient to use the purely complex description of the quiver variety given in $\S 1.1$. In the case of a simple reflection $s_{a}$, the construction is as follows. Let $(B, i, j) \in \mathbf{M}_{\zeta}^{s s}(\mathbf{v}, \mathbf{w})$. Define vector spaces $V_{k}^{\prime}, W_{k}^{\prime}$ by $V_{k}^{\prime}=V_{k}$ if $k \neq a$,

$$
V_{a}^{\prime}=\left(W_{a} \oplus \bigoplus_{o(h)=a} V_{i(h)}\right) /\left(j_{a}+\sum_{o(h)=a} B_{h}\right) V_{a}
$$

and $W_{k}^{\prime}=W_{k}$ for all $k$. Let $Z$ be the set of all $\left(B^{\prime}, i^{\prime}, j^{\prime}\right) \in \mathbf{M}_{s_{a}}\left(\mathbf{v}^{\prime}, \mathbf{w}^{\prime}\right)$ such that:

(i) $B_{h}^{\prime}=B_{h}$ if $i(h) \neq a$ and $o(h) \neq a$;

(ii) $i_{k}=i_{k}^{\prime}$ and $j_{k}^{\prime}=j_{k}$ if $k \neq a$;

(iii) set

$$
\begin{gathered}
x_{a}=j_{a} \oplus \bigoplus_{o(h)=a} B_{h}: V_{a} \rightarrow W_{a} \oplus \bigoplus_{o(h)=a} V_{i(h)}, \\
y_{a}=i_{a} \oplus \bigoplus_{i(h)=a} \epsilon(\bar{h}) B_{h}: W_{a} \oplus \bigoplus_{i(h)=a} V_{o(h)} \rightarrow V_{a}
\end{gathered}
$$

and define $x_{a}^{\prime}$ and $y_{a}^{\prime}$ in a similar fashion. The sequence

$$
0 \rightarrow V_{a} \stackrel{x_{a}}{\longrightarrow} W_{a} \oplus \bigoplus_{o(h)=a} V_{i(h)} \stackrel{y_{b}^{\prime}}{\longrightarrow} V_{a}^{\prime} \rightarrow 0
$$

is exact, and $x_{a} y_{a}=x_{a}^{\prime} y_{a}^{\prime}-\lambda_{a} \mathrm{Id}$.

Then (see [Maf02]), $Z$ is a principal $G L\left(V_{a}\right)$-homogeneous space. Thus, it corresponds to a unique point $\kappa_{s_{a}}(B, i, j) \in \mathcal{M}_{s_{a} \zeta}\left(\mathbf{v}^{\prime}, \mathbf{w}^{\prime}\right)=\mathcal{M}_{s_{a} \zeta}\left(s_{a} \cdot(\mathbf{v}, \mathbf{w})\right)$.

\section{Categories of $(A, c)$-complexes}

2.1 Let $A$ be a $\mathbb{Z}$-graded ring and $c$ a central element of $A$ of degree 2. We denote by $Z_{2}(A)$ the degree 2 summand of the center of $A$, so that $c \in Z_{2}(A)$.

Definition 1. A left $(A, c)$-complex is a $\mathbb{Z}$-graded left $A$-module $M$ together with a degree one map $d: M \rightarrow M$ such that

$$
d^{2}=c
$$

and $d$ (super)commutes with the action of $A$ :

$$
d(a m)=(-1)^{|a|} a d(m), \quad a \in A, m \in M .
$$

A morphism of $(A, c)$-complexes is a degree zero morphism of $A$-modules which commutes with $d$. The category $\operatorname{Com}(A, c)$ of left $(A, c)$-complexes is abelian. The translation functor [1] in the category $\operatorname{Com}(A, c)$ is defined by

$$
(M[1])^{i}=M^{i+1}, \quad d_{[1]}=-d,
$$

and the $A$-module structure on $M[1]$ is

$$
a \circ m=(-1)^{|a|} a m .
$$




\section{Frenkel, M. Khovanov and O. Schiffmann}

Alternatively, we may define a $\mathbb{Z}$-graded algebra $\widetilde{A}_{c}=A \dot{\otimes} \mathbb{C}[d] /\left(d^{2}-c\right)$ with $d a=(-1)^{|a|} a d$ and $\operatorname{deg}(d)=1$. Then $\operatorname{Com}(A, c)$ is nothing but the category $\operatorname{Mod}\left(\widetilde{A}_{c}\right)$ of graded left $\widetilde{A}_{c}$-modules.

2.2 Let $M$ and $N$ be left $(A, c)$-complexes. Given a morphism of graded $A$-modules $h: M \rightarrow N[-1]$, the map $f=h d_{M}+d_{N} h$ is a morphism $M \rightarrow N$ of $(A, c)$-complexes. Any such morphism is called null-homotopic. The following result is clear.

Proposition 2.1. Null-homotopic morphisms form a two-sided ideal in the category $\operatorname{Com}(A, c)$.

We say that morphisms $f, g: M \rightarrow N$ are homotopic and write $f \sim g$ if $f-g$ is null-homotopic. Define the homotopy category $\mathcal{K}(A, c)$ as follows. Objects are $(A, c)$-complexes and for any two (A,c)-complexes $M$ and $N$ we put

$$
\operatorname{Hom}_{\mathcal{K}(A, c)}(M, N)=\operatorname{Hom}_{\mathbf{C o m}(A, c)}(M, N) / \sim .
$$

Categories $\operatorname{Com}(A, c)$, as well as $\mathcal{K}(A, c)$, for various $c \in Z_{2}(A)$, might have common objects. If $M \in \operatorname{Com}(A, c)$ and $c^{\prime} M=0$ for some $c^{\prime} \in Z_{2}(A)$ then $M \in \operatorname{Com}\left(A, c+c^{\prime}\right)$.

\subsection{Tensor product of left and right $(A, c)$-complexes}

If $M$ is a right graded $A$-module and $N$ a left graded $A$-module, the tensor product $M \otimes_{A} N$ is a graded abelian group. If $M$ is a right $(A, c)$-complex and $N$ a left $(A,-c)$-complex, then $M \otimes_{A} N$ is a complex of graded abelian groups with the differential

$$
d(m \otimes n)=d m \otimes n+(-1)^{|m|} m \otimes d n,
$$

since

$$
d^{2}(m \otimes n)=d^{2} m \otimes n+m \otimes d^{2} n=m c \otimes n+m \otimes(-c) n=0 .
$$

\subsection{Bimodules}

Let $c_{0}, c_{1} \in Z_{2}(A)$. An $\left(A, c_{0}, c_{1}\right)$-complex is a graded $A$-bimodule $N$ together with a degree one map $d: N \rightarrow N$ such that $d^{2}=l_{c_{0}}+r_{c_{1}}$, (where $l_{c_{0}}$ is left multiplication by $c_{0}$ and $r_{c_{1}}$ is right multiplication by $\left.c_{1}\right), d$ (super)commutes with the left action of $A$ :

$$
d(a n)=(-1)^{|a|} a d n, \quad a \in A, n \in N,
$$

and commutes with the right action of $A$.

If $M$ is a left $\left(A,-c_{1}\right)$-complex, the tensor product $N \otimes_{A} M$ is a left $\left(A, c_{0}\right)$-complex. Thus, the tensor product with $N$ is a functor from $\operatorname{Com}\left(A,-c_{1}\right)$ to $\operatorname{Com}\left(A, c_{0}\right)$, and from $\mathcal{K}\left(A,-c_{1}\right)$ to $\mathcal{K}\left(A, c_{0}\right)$.

\section{Categories of duplexes}

3.1 Let $A$ be a $\mathbb{Z} / 2 \mathbb{Z}$-graded ring, $A=A_{0} \oplus A_{1}$, and $c$ a degree zero central element of $A$. A duplex over $(A, c)$ is a $\mathbb{Z} / 2 \mathbb{Z}$-graded $A$-module $M=M^{0} \oplus M^{1}$ with a generalized differential

$$
M^{0} \stackrel{d}{\longrightarrow} M^{1} \stackrel{d}{\longrightarrow} M^{0}
$$

which supercommutes with the action of $A$ and satisfies $d^{2}(m)=\mathrm{cm}$ for all $m \in M$. A duplex over $(A, 0)$ is simply a 2-periodic complex of $A$-modules. An $(A, c)$-duplex for $c \neq 0$ can be viewed as a 2-periodic 'complex' with the differential satisfying $d^{2}=c$ rather than $d^{2}=0$. 


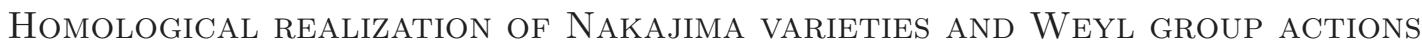

A homomorphism $f: M \rightarrow N$ of $(A, c)$-duplexes is a degree zero $A$-module map that commutes with the differentials:

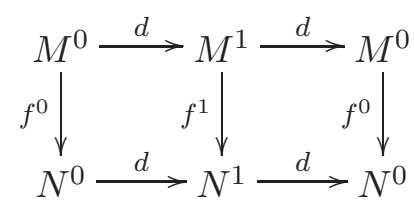

We denote by $\mathbf{C o m}_{2}(A, c)$ the category of $(A, c)$-duplexes. The shift functor [1] in this category is 2-periodic, $[2] \cong \mathrm{Id}$. The category $\mathbf{C o m}_{2}(A, c)$ is abelian. We will often write duplexes in the form

$$
\stackrel{d}{\longrightarrow} M^{0} \stackrel{d}{\longrightarrow} M^{1} \stackrel{d}{\longrightarrow}
$$

Given maps $f: M \rightarrow N$ and $g: N \rightarrow M$ of $(A, c)$-duplexes such that $f g=c_{1}$ and $g f=c_{1}$ for some degree zero central element $c_{1}$ of $A$,

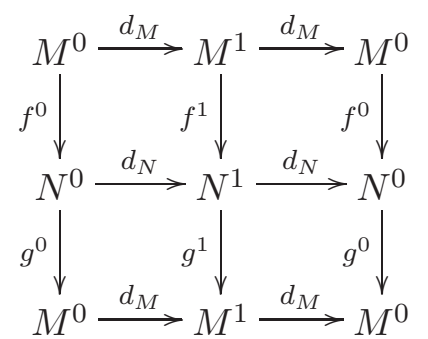

the cone of $(f, g)$ is defined as the 'total' $\left(A, c+c_{1}\right)$-duplex of the above diagram,

$$
\stackrel{d}{\longrightarrow} M^{0} \oplus N^{1} \stackrel{d}{\longrightarrow} M^{1} \oplus N^{0} \stackrel{d}{\longrightarrow}
$$

with $d=d_{M}-d_{N}+f+g$.

We will also use a less precise notation $\stackrel{g}{\longrightarrow} M \stackrel{f}{\longrightarrow} N \stackrel{g}{\longrightarrow}$ for the cone of $(f, g)$.

Note that we may once again think of $\operatorname{Com}_{2}(A, c)$ as the category of $\mathbb{Z} / 2 \mathbb{Z}$-graded left $\widetilde{A}_{c}$-modules, where $\widetilde{A}_{c}$ is defined as in $\S 2$.

3.2 Given an $A$-homomorphism $h: M \rightarrow N[-1]$, the map $f=h d_{M}+d_{N} h$ is a morphism $M \rightarrow N$ of duplexes. We will say that morphisms $f, g: M \rightarrow N$ are homotopic if $f-g=h d_{M}+d_{N} h$ for some $h$. The following is straightforward.

Proposition 3.1. Null-homotopic morphisms form a two-sided ideal in the category $\mathbf{C o m}_{2}(A, c)$.

We call the quotient category of $\mathbf{C o m}_{2}(A, c)$ by this ideal the homotopy category of $(A, c)$-duplexes and denote it by $\mathcal{K}_{2}(A, c)$.

Example. For an $A$-module $M$ let $M_{c, 1}$ be the duplex

$$
\stackrel{1}{\longrightarrow} M \stackrel{c}{\longrightarrow} M \stackrel{1}{\longrightarrow}
$$

which is the cone of $(c, 1)$. The identity morphism of $M_{c, 1}$ is null-homotopic, and $M_{c, 1}$ is isomorphic to the zero object in the homotopy category of duplexes.

Remark. If $c$ is invertible, any $(A, c)$-duplex is trivial in the homotopy category, and the category $\mathcal{K}_{2}(A, c)$ is trivial. The case of noninvertible $c$ is more interesting. If $A$ is artinian, any element of $A$ is either invertible or nilpotent, and the only nontrivial case is that of nilpotent $c$.

Proposition 3.2. If $M$ is an $(A, c)$-duplex and $I$ an injective $\mathbb{Z} / 2 \mathbb{Z}$-graded $A$-submodule of $M$ such that $d$ is injective on $I$ and $I \cap d I=0$, then $M$ is isomorphic in the homotopy category to its quotient by the subduplex generated by $I$ :

$$
M \cong\left\{\longrightarrow M^{0} /\left(I^{0} \oplus d\left(I^{1}\right)\right) \longrightarrow M^{1} /\left(I^{1} \oplus d\left(I^{0}\right)\right) \longrightarrow\right\} .
$$




\section{Frenkel, M. Khovanov and O. Schiffmann}

The proof is again straightforward.

\subsection{Duplexes of bimodules}

Tensor product with a duplex $N$ of $A$-bimodules such that $d^{2}=l_{c_{0}}+r_{c_{1}}$ is a functor from $\operatorname{Com}_{2}\left(A,-c_{1}\right)$ to $\mathbf{C o m}_{2}\left(A, c_{0}\right)$ and from $\mathcal{K}_{2}\left(A,-c_{1}\right)$ to $\mathcal{K}_{2}\left(A, c_{0}\right)$.

\section{Stable categories}

4.1 Let $A$ be a $\mathbb{Z}$-graded ring and $c \in Z_{2}(A)$ a degree two central element. Let $\underline{\operatorname{Mod}}(A)$ denote the stable category of $\mathbb{Z}$-graded $A$-modules (see, e.g., [Hap88]). Its objects are $\mathbb{Z}$-graded $A$-modules and for any modules $M$ and $N$ we have

$$
\operatorname{Hom}_{\underline{\operatorname{Mod}}(A)}(M, N)=\operatorname{Hom}_{A}(M, N) / I
$$

where $I$ is the ideal of all morphisms $f: M \rightarrow N$ which admit a factorization $f=g \circ h$ where $h: M \rightarrow P, g: P \rightarrow N$ for some projective module $P$. In particular, an object $M$ of $\underline{\operatorname{Mod}}(A)$ is isomorphic to the zero object if and only if it is projective as an $A$-module. We define the stable category $\underline{\operatorname{Mod}}\left(\widetilde{A}_{c}\right)$ in a same way. Since $\widetilde{A}_{c}$ is projective (in fact, free) as an $A$-module, there is a natural restriction functor $R: \underline{\operatorname{Mod}}\left(\widetilde{A}_{c}\right) \rightarrow \underline{\operatorname{Mod}}(A)$.

There is a canonical functor $\Phi: \operatorname{Mod}\left(\widetilde{A}_{c}\right) \simeq \operatorname{Com}(A, c) \rightarrow \mathcal{K}(A, c)$.

Lemma 4.1. For any projective $\widetilde{A}_{c}$-module $P$ we have $\Phi(P)=0$.

Proof. Let $\left\{P_{i}^{\prime}\right\}$ be the collection of indecomposable projective $A$-modules. It is easy to check that $P_{i}=\widetilde{A}_{c} \otimes_{A} P_{i}^{\prime}$ is an indecomposable projective $\widetilde{A}_{c}$-module, and hence that $\left\{P_{i}\right\}$ forms the complete collection of indecomposable projectives for $\widetilde{A}_{c}$. However, $P_{i}=P_{i}^{\prime} \oplus P_{i}^{\prime}[-1]$ as $A$-module and $d: P_{i}^{\prime} \stackrel{\sim}{\rightarrow} P_{i}^{\prime}[-1]$, so that $P_{i}$ is homotopic to zero as an $(A, c)$-complex.

We deduce that the functor $\Phi$ admits a factorization

$$
\operatorname{Mod}\left(\widetilde{A}_{c}\right) \simeq \operatorname{Com}(A, c) \stackrel{\Phi_{1}}{\longrightarrow} \underline{\operatorname{Mod}}\left(\widetilde{A}_{c}\right) \stackrel{\Phi_{2}}{\longrightarrow} \mathcal{K}(A, c) .
$$

Similar results hold for $\mathbf{C o m}_{2}(A, c), \underline{\operatorname{Mod}}_{2}\left(\widetilde{A}_{c}\right)$ and $\mathcal{K}_{2}(A, c)$ if $A$ is a $\mathbb{Z} / 2 \mathbb{Z}$-graded ring.

4.2 Proposition 3.2 admits the following straightforward generalization.

Proposition 4.1. If $M$ is an $(A, c)$-duplex and $I$ an injective and projective $\mathbb{Z} / 2 \mathbb{Z}$-graded $A$-submodule of $M$ such that $d$ is injective on $I$ and $I \cap d I=0$, then $M$ is isomorphic in the stable category $\underline{\operatorname{Mod}}_{2}\left(\widetilde{A}_{c}\right)$ to its quotient by the subduplex generated by $I$ :

$$
M \cong\left\{\longrightarrow M^{0} /\left(I^{0} \oplus d\left(I^{1}\right)\right) \longrightarrow M^{1} /\left(I^{1} \oplus d\left(I^{0}\right)\right) \longrightarrow\right\} .
$$

\section{Homological realization of Nakajima varieties}

5.1 Let $\mathbf{Q}=(I, E), H$ and $\epsilon$ be as in $\S$ 1.1. We can view $(I, H)$ as the oriented double of the unoriented graph $\mathbf{Q}$. Consider the path algebra of $(I, H)$. Note that in this algebra the product $h h^{\prime}$ of two length one paths is nonzero if and only if $i(h)=o\left(h^{\prime}\right)$.

Define the $\mathbb{C}$-algebra $A(\mathbf{Q})$ as the quotient of this path algebra by relations:

(i) $h h^{\prime}=0$ if $h^{\prime} \neq \bar{h}$;

(ii) $\epsilon(h) h \bar{h}=\epsilon\left(h^{\prime}\right) h^{\prime} \overline{h^{\prime}}$ if $o(h)=o\left(h^{\prime}\right)$.

Relations of the second type say that $\epsilon(h) h \bar{h}$ in the quotient algebra depends only on the outgoing vertex of $h$. We denote $X_{a}=\epsilon(h) h \bar{h}$ where $a=o(h)$. 


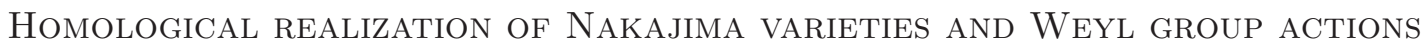

If the graph has only two vertices, $a$ and $b$, and one edge connecting them, we let $A(\mathbf{Q})$ be the quotient of the path algebra by relations $h \bar{h} h=0=\bar{h} h \bar{h}$ (where $h$ is one of the orientations of the edge and $\bar{h}$ is the reverse of $h$ ). If the graph has only one vertex $a$, and no edges, define $A(\mathbf{Q})$ as the exterior algebra on one generator $X_{a}$, and place it in degree 2 to make $A(\mathbf{Q})$ graded. If the graph has more than one vertex, we grade $A(\mathbf{Q})$ by lengths of paths. The graded algebra $A(\mathbf{Q})$ is (up to isomorphism) independent of the choice of the orientation $\epsilon$.

For simplicity, we will write $A$ instead of $A(\mathbf{Q})$. The algebra $A$ is finite-dimensional, $\operatorname{dim}(A)=$ $2(|I|+|E|)$. Any path of length at least 3 equals 0 in $A$.

Note that $X_{a}$ (see above) is central, and $X_{a}$, over all vertices $a$, form a basis for the degree 2 subspace of $A$.

A length 0 path $(a)$, for a vertex $a \in I$, is a minimal idempotent in $A$, and $1=\sum_{a}(a)$.

Example. If $\mathbf{Q}$ has one vertex and one loop, $A$ is isomorphic to the exterior algebra on two generators $h, \bar{h}$ :

$$
A \cong \mathbb{C}\langle h, \bar{h}\rangle / h^{2}=\bar{h}^{2}=h \bar{h}+\bar{h} h=0 .
$$

The trace $\operatorname{tr}: A \rightarrow \mathbb{C}$ defined by

$$
\operatorname{tr}\left(X_{a}\right)=1, \quad \operatorname{tr}(h)=\operatorname{tr}((a))=0,
$$

makes $A$ into a graded Frobenius algebra. Note that $A$ is symmetric (but with respect to a different trace) if and only if $\mathbf{Q}$ is bipartite (i.e. if it is possible to partition the set of vertices of $\mathbf{Q}$ into two disjoint sets in such a way that all edges go from one set to the other). In the latter case, $A$ is isomorphic to the zigzag algebra of $\mathbf{Q}$, see [HK01]. For any $\mathbf{Q}$, the algebra $A$ is a skew-zigzag algebra, in the terminology of [HK01, § 4.6].

Denote by $P_{a}$ the indecomposable projective left $A$-module $A(a)$. An indecomposable projective left $A$-module is isomorphic to $P_{a}$, for some $a$. Denote by ${ }_{a} P$ the indecomposable projective right $A$-module $(a) A$. Since $A$ is Frobenius, $P_{a}$ and ${ }_{a} P$ are, in addition, injective.

Let $S_{a}$ be the simple quotient of $P_{a}$ (equivalently, the quotient of $P_{a}$ by all paths of length greater than 0$)$. Denote by $\widehat{a}$ the image of $(a) \in P_{a}$ under the quotient map. $S_{a}$ is a one-dimensional complex vector space and is spanned by $\widehat{a}$. A simple left $A$-module is isomorphic to $S_{a}$, for some $a$. The modules $P_{a}, S_{a}$, and ${ }_{a} P$ inherit $\mathbb{Z}$-grading from $A$.

Denote by $[m]$ the grading shift down by $m$., i.e $(M[m])^{l}=M^{m+l}$. Let $\mathbf{M o d}(A)(\operatorname{respec}-$ tively $\operatorname{HMod}(A))$ be the category of graded $A$-modules (respectively the category of graded $A$-modules equipped with a Hermitian structure $x \mapsto x^{*}$ such that $h\left(x^{*}\right)=(h x)^{*}$ for all edges $h$ ). For any two graded $A$-modules $M, N$ we denote by $\operatorname{Hom}_{A}(M, N)$ the set of grading-preserving A-homomorphisms.

The modules $P_{a},{ }_{a} P, S_{a}$ have unique Hermitian structures $x \mapsto x^{*}$ such that $(a)^{*}=(a)$ and $h\left(x^{*}\right)=(h x)^{*}$ for all edges $h$.

5.2 Let $V_{a}, W_{a}, a \in I$ be finite-dimensional $\mathbb{C}$-vector spaces. Consider the graded $A$-module

$$
M=\bigoplus_{a} V_{a} \otimes P_{a} \oplus W_{a} \otimes S_{a}[-1],
$$

a direct sum of a projective and a semisimple $A$-module. We raise the grading of simple modules $S_{a}$ by 1 to 'balance' them in the middle of projective modules $P_{a}$, the latter non-zero in degrees $0,1,2$.

Let us equip $M$ with a degree 1 generalized differential $d: M \rightarrow M$ with the property $d^{2}=c$ for a fixed degree 2 central element $c$ of $A$,

$$
c=\sum_{a} c_{a} X_{a}, \quad c_{a} \in \mathbb{C} .
$$




\section{Frenkel, M. Khovanov and O. Schiffmann}

$d$ should super-commute with $A$,

$$
d x=(-1)^{|x|} x d, \quad x \in A .
$$

The graded components of $M$ are

$$
\begin{aligned}
& M^{0}=\bigoplus_{a}\left(V_{a} \otimes(a)\right), \\
& M^{1}=\bigoplus_{a}\left(\left(W_{a} \otimes \widehat{a}\right) \bigoplus_{o(h)=a}\left(V_{i(h)} \otimes h\right)\right), \\
& M^{2}=\bigoplus_{a}\left(V_{a} \otimes X_{a}\right),
\end{aligned}
$$

and the differential must have the form

$$
0 \longrightarrow M^{0} \stackrel{d^{0}}{\longrightarrow} M^{1} \stackrel{d^{1}}{\longrightarrow} M^{2} \longrightarrow 0 .
$$

Since $d(a)=(a) d$, for minimal idempotents $(a) \in A$, the generalized complex decomposes into the sum of

$$
0 \longrightarrow(a) M^{0} \stackrel{d^{0}}{\longrightarrow}(a) M^{1} \stackrel{d^{1}}{\longrightarrow}(a) M^{2} \longrightarrow 0,
$$

over all $a$. We can write the latter as

$$
0 \longrightarrow V_{a} \otimes(a) \stackrel{d^{0}}{\longrightarrow} \bigoplus_{o(h)=a}\left(V_{i(h)} \otimes h\right) \oplus\left(W_{a} \otimes \widehat{a}\right) \stackrel{d^{1}}{\longrightarrow} V_{a} \otimes X_{a} \longrightarrow 0 .
$$

The components of $d^{0}$ can be described as maps $B_{h} \in \operatorname{Hom}\left(V_{a}, V_{i(h)}\right), j_{a} \in \operatorname{Hom}\left(V_{a}, W_{a}\right)$ :

$$
d^{0}=\left(\bigoplus_{o(h)=a} B_{h}, j_{a}\right)^{\mathrm{t}}
$$

The superscript $\mathrm{t}$ in the formula stands for transposing a row vector into a column vector. From $d h=-h d$, for all edges $h$, we derive that

$$
d^{1}=\left(\bigoplus_{o(h)=a} \epsilon(\bar{h}) B_{\bar{h}}, i_{a}\right)
$$

where $i_{a} \in \operatorname{Hom}\left(W_{a}, V_{a}\right)$. We should have $c=d^{1} d^{0}$, or, specializing to a vertex $a$,

$$
c_{a} \mathrm{Id}=\sum_{o(h)=a} \epsilon(\bar{h}) B_{\bar{h}} B_{h}+i_{a} j_{a} .
$$

The right-hand side is the a-component of the complex moment map for the Nakajima quiver varieties.

If $d$ is given as above by the data $d=\left(B_{h}, i_{a}, j_{a}\right)$ we may define its Hermitian adjoint $d^{*}=$ $\left(\epsilon(\bar{h}) B_{\bar{h}}^{*},-j_{a}^{*}, i_{a}^{*}\right)$ to be of the same form. The real component of the moment map equation $\mu_{\mathbb{R}}(B, i, j)=\zeta_{\mathbb{R}}$ is equivalent to the relation

$$
\frac{\sqrt{-1}}{2}\left(d d^{*}+d^{*} d\right)=\sum_{a} \zeta_{\mathbb{R}, a} X_{a}
$$

We can think of $\boldsymbol{\zeta}_{\mathbb{C}}$ and $\boldsymbol{\zeta}_{\mathbb{R}}$ as degree 2 central elements of $A$, by taking the standard bases of $\mathbb{C}^{I}$ and $\mathbb{R}^{I}$ to $\left\{X_{a}\right\}_{a \in I}$. Collapse the grading from $\mathbb{Z}$ to $\mathbb{Z} / 2 \mathbb{Z}$, and write $\boldsymbol{M o d}_{2}(A)$ and $\mathbf{H M o d}_{2}(A)$ for the corresponding categories of $\mathbb{Z} / 2 \mathbb{Z}$-graded modules. Equations (5.1) and (5.2) together with the definitions of quiver varieties imply the following result. 


\section{Homological REALIZATION OF NAKAJIMA VARIETIES AND WEYL GROUP ACTIONS}

Proposition 5.1. For any $\zeta_{\mathbb{C}}, \zeta_{\mathbb{R}}$ there is a bijection between points on the Nakajima variety $\mathcal{M}_{\zeta}(\mathbf{v}, \mathbf{w})$ and isomorphism classes of the following data $(M, d, \psi)$ :

$A$ graded $A$-module $M \in \mathbf{H M o d}_{2}(A)$ which is a direct sum of a projective and a semisimple module, with $v_{a}$ the multiplicity of $P_{a}$ and $w_{a}$ the multiplicity of $S_{a}[-1]$, with a generalized differential $d$ such that $d^{2}=\boldsymbol{\zeta}_{\mathbb{C}}$, and $(\sqrt{-1} / 2)\left(d d^{*}+d^{*} d\right)=\boldsymbol{\zeta}_{\mathbb{R}}$, and isomorphisms $\psi_{a}: W_{a} \cong \operatorname{Hom}_{A}\left(S_{a}[-1], M\right)$.

Now suppose that $\zeta_{\mathbb{C}}$ is generic. The complex description of quiver varieties yield the following result.

Proposition 5.2. There is a bijection between points on the Nakajima variety $\mathcal{M}_{\zeta}(\mathbf{v}, \mathbf{w})$ and isomorphism classes of the following data $(M, d, \psi)$.

$A$ graded $A$-module $M \in \operatorname{Mod}_{2}(A)$ which is a direct sum of a projective and a semisimple module, with $v_{a}$ the multiplicity of $P_{a}$ and $w_{a}$ the multiplicity of $S_{a}[-1]$, with a generalized differential $d$ such that $d^{2}=\boldsymbol{\zeta}_{\mathbb{C}}$, and isomorphisms $\psi_{a}: W_{a} \cong \operatorname{Hom}_{A}\left(S_{a}[-1], M\right)$.

It is easy to check that two nonisomorphic data $(M, d, \psi)$ as above remain nonisomorphic after applying the functor $\Phi_{1}$ (see (4.1)), and that

$$
\operatorname{Hom}_{A}\left(S_{a}[-1], M\right) \cong \operatorname{Hom}_{\underline{\operatorname{Mod}}_{2}(A)}\left(S_{a}[-1], R \Phi_{1}(M)\right)
$$

for any $M$ as above. This gives the following variant of Proposition 5.2.

Proposition 5.3. There is a bijection between points on the Nakajima variety $\mathcal{M}_{\zeta}(\mathbf{v}, \mathbf{w})$ and isomorphism classes of the following data $(\underline{M}, \psi)$.

An object $\underline{M} \in \underline{\operatorname{Mod}}_{2}\left(\widetilde{A}_{\zeta_{\mathbb{C}}}\right)$ such that $\underline{M} \simeq \Phi_{1}\left(M^{\prime}\right)$ for some $M^{\prime} \in \operatorname{Com}_{2}\left(A, \boldsymbol{\zeta}_{\mathbb{C}}\right)$ with $M^{\prime} \simeq$

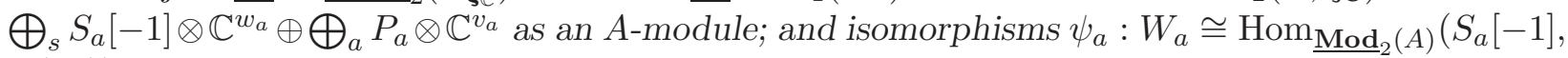
$R(\underline{M}))$.

Consider now the case when $\zeta_{\mathbb{C}}=0$ and $\zeta_{\mathbb{R}} \in\left(\mathbb{N}^{+}\right)^{I}$. The description of $\mathbf{M}_{\zeta}^{s s}(\mathbf{v}, \mathbf{w})$ given in $\S 1.1$ case (i) yields the following.

Proposition 5.4. There is a bijection between points on $\mathcal{M}_{\zeta}(\mathbf{v}, \mathbf{w})$ and isomorphism classes of $(M, d, \psi)$ where $M \in \operatorname{Mod}_{2}(A)$ and $\psi$ are as in Proposition 5.2, $d^{2}=0$ and no projective submodule of $M$ is $d$-stable.

Remark. All the above results also hold in the $\mathbb{Z}$-graded case.

\section{Weyl group action in categories of duplexes}

6.1 We use notation from $\S 5.1$. Choose a vertex $a$ without a loop. Since $P_{a}$ is a left $A$-module and ${ }_{a} P$ a right $A$-module, $P_{a} \otimes{ }_{a} P$ is an $A$-bimodule (the tensor product is over $\mathbb{C}$ ). Denote by $m_{a}: P_{a} \otimes{ }_{a} P \longrightarrow A$ the bimodule map which is simply the restriction of the multiplication map $A \otimes A \longrightarrow A$, so that $m_{a}((a) \otimes(a))=(a)$. Denote by $\Delta_{a}: A \longrightarrow P_{a} \otimes{ }_{a} P$ the bimodule map determined by

$$
\Delta_{a}(1)=X_{a} \otimes(a)+(a) \otimes X_{a}+\sum_{o(h)=a} \epsilon(h) \bar{h} \otimes h,
$$

the sum over all edges $h$ that start at $a$.

6.2 Let $C_{a, x}$, for $x \in \mathbb{C}$, be the following duplex of bimodules:

$$
\stackrel{\Delta_{a}}{\longrightarrow} P_{a} \otimes{ }_{a} P \stackrel{x m_{a}}{\longrightarrow} A \stackrel{\Delta_{a}}{\longrightarrow} .
$$




\section{Frenkel, M. Khovanov and O. Schiffmann}

If $x \neq 0$, this duplex is isomorphic to

$$
\stackrel{x \Delta_{a}}{\longrightarrow} P_{a} \otimes{ }_{a} P \stackrel{m_{a}}{\longrightarrow} A \stackrel{x \Delta_{a}}{\longrightarrow} .
$$

Denote by $d$ the differential in (6.1) and (6.2). We have

$$
\begin{aligned}
& \left.d^{2}\right|_{P_{a} \otimes{ }_{a} P}=x\left(X_{a} \otimes 1+1 \otimes X_{a}\right), \\
& \left.d^{2}\right|_{A}=x\left(2 X_{a}-\sum_{o(h)=a} X_{i(h)}\right) .
\end{aligned}
$$

Here $X_{a} \otimes 1$ is the left multiplication by $X_{a}$, etc. Hence, as operators on $C_{a, x}$ we have

$$
d^{2}=x\left(X_{a} \otimes 1+1 \otimes X_{a}-\sum_{o(h)=a} X_{i(h)} \otimes 1\right) .
$$

Note that $X_{b} \otimes 1-1 \otimes X_{b}$ acts trivially on $P_{a} \otimes{ }_{a} P$ and $A$ if $a \neq b$. Thus, we also have

$$
d^{2}=x\left(X_{a} \otimes 1+1 \otimes X_{a}-\sum_{o(h)=a} X_{i(h)} \otimes 1\right)+\sum_{b \neq a} x_{b}\left(X_{b} \otimes 1-1 \otimes X_{b}\right)
$$

for any $x_{b} \in \mathbb{C}$, as $b$ ranges over all vertices other than $a$.

The Weyl group $\mathbf{W}$ of $(I, E)$ has generators $s_{a}$, over all loopless vertices $a \in I$, and relations:

(i) $s_{a}^{2}=1$ for all $a$;

(ii) $s_{a} s_{b}=s_{a} s_{b}$ if $a$ and $b$ do not have a common edge;

(iii) $s_{a} s_{b} s_{a}=s_{b} s_{a} s_{b}$ if $a$ and $b$ are joined by exactly one edge.

The Weyl group acts on $Z_{2}(A)$, the degree 2 summand of the center of $A$ (on the vector space with the basis $\left.\left\{X_{a}\right\}_{a \in I}\right)$ by

$$
s_{a}(c)=c+x_{a}\left(\sum_{o(h)=a} X_{i(h)}-2 X_{a}\right)
$$

for $c=\sum_{b \in I} x_{b} X_{b}$. This action is compatible with the one defined on $\zeta_{\mathbb{C}}$ in $\S 1.4$. via the natural identification $\mathbb{C}^{I} \rightarrow Z_{2}(A)$. It follows from (6.4) that, for any $c \in Z_{2}(A)$, the tensor product with $C_{a,-x_{a}}$ is a functor from $\mathbf{C o m}_{2}(A, c)$ to $\mathbf{C o m}_{2}\left(A, s_{a}(c)\right)$ and from $\mathcal{K}_{2}(A, c)$ to $\mathcal{K}_{2}\left(A, s_{a}(c)\right)$. Denote this functor by $\mathcal{R}_{a}: \mathcal{K}_{2}(A, c) \rightarrow \mathcal{K}_{2}\left(A, s_{a}(c)\right)$.

Lemma 6.1. The functor $\mathcal{R}_{a}$ lifts to a functor from $\underline{\operatorname{Mod}}_{2}\left(\widetilde{A}_{c}\right)$ to $\underline{\operatorname{Mod}}_{2}\left(\widetilde{A}_{s_{a}(c)}\right)$.

Proof. Let $\tilde{P}_{b}=\widetilde{A}_{c} \otimes_{A} P_{b}$ be an indecomposable projective $\widetilde{A}_{c}$-module. We have to show that $C_{a,-x_{a}} \otimes \tilde{P}_{b}$ is a projective $\widetilde{A}_{s_{a}(c)}$-module. By definition, we have

$$
C_{a, x} \otimes \tilde{P}_{b}=\left(\begin{array}{c}
A \otimes \tilde{P}_{b} \\
\oplus \\
\left(P_{a} \otimes{ }_{a} P\right)[1] \otimes_{A} \tilde{P}_{b}
\end{array}\right)=\left(\begin{array}{c}
P_{b} \oplus P_{b}[1] \\
\oplus \\
\underset{\substack{\oplus \\
o(h)=a \\
i(h)=b}}{\oplus}\left(P_{a}[1] \oplus P_{a}\right)
\end{array}\right),
$$

and the action of the element $d \in A_{s_{a}(c)}$ is given by

$$
d=\left(\begin{array}{cc}
1 \otimes d & m_{a} \otimes 1 \\
\Delta_{a} \otimes 1 & 1 \otimes d
\end{array}\right) .
$$

Since $A$ is Frobenius, $P_{a}$ and $P_{b}$ are both projective and injective. Furthermore, it is easy to check that $d$ is injective on $P_{b}$ and that $P_{b} \cap d\left(P_{b}\right)=0$. Similarly, we have $d$ is injective on $P_{a}[1]$ and we have $P_{a}[1] \cap d\left(P_{a}[1]\right)=0$. By applying Proposition 4.1 twice we see that $C_{a, x} \otimes \tilde{P}_{b}=0$ in $\underline{\operatorname{Mod}}_{2}\left(\widetilde{A}_{s_{a}(c)}\right)$ as desired. 


\section{Homological ReAlization of NAKajima varieties AND Weyl group aCtions}

We will denote this functor by the same symbol $\mathcal{R}_{a}: \underline{\operatorname{Mod}}_{2}\left(\widetilde{A}_{c}\right) \rightarrow \underline{\operatorname{Mod}}_{2}\left(\widetilde{A}_{s_{a}(c)}\right)$.

6.3 We now deal with the categorical analogue of the braid relation.

Proposition 6.1. If $x \neq 0$, there is an isomorphism in the stable category of bimodule duplexes

$$
C_{a,-x} \otimes_{A} C_{a, x} \cong A .
$$

Proof. Let $N=C_{a,-x} \otimes_{A} C_{a, x}$ and $\partial$ be the differential in $N$. Note that $\partial^{2}=0$, and $N$ is a duplex of $A$-bimodules.

Since $x \neq 0$, we can write $C_{a,-x}$ as

$$
\stackrel{\Delta_{a}}{\longrightarrow} P_{a} \otimes{ }_{a} P \stackrel{-x m_{a}}{\longrightarrow} A \stackrel{\Delta_{a}}{\longrightarrow}
$$

$N$ is the total duplex of the following diagram (which is 2-cyclic in horizontal and vertical directions, and each of the four squares anticommutes)

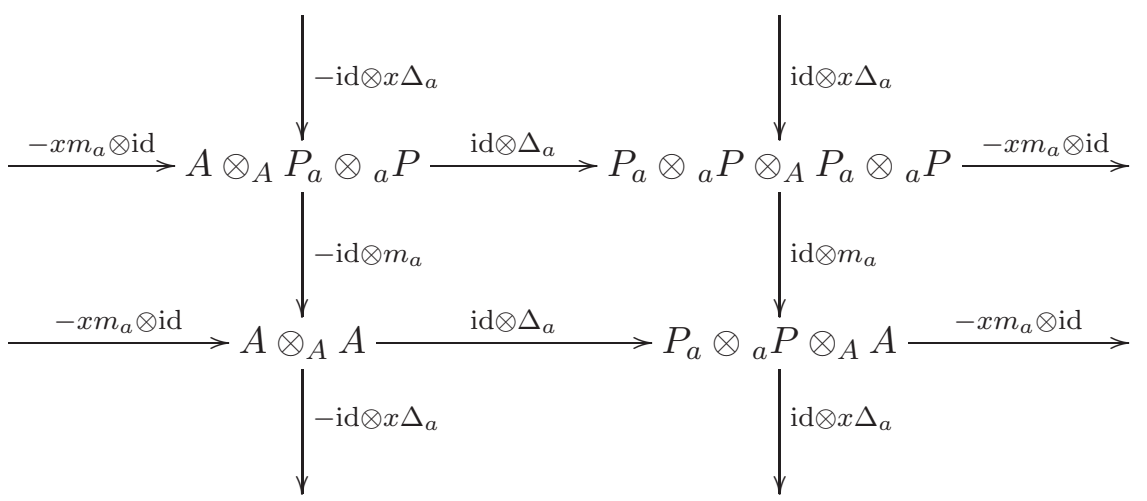

Denote by $N_{i j}$ the four bimodules in the above diagram:

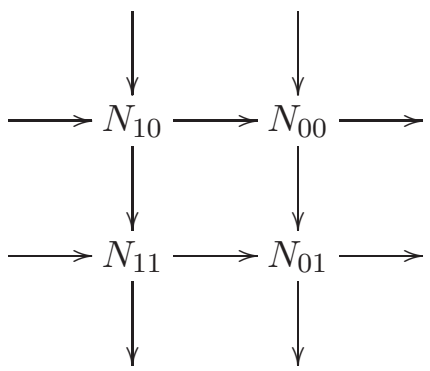

Simplifying our notation as at the end of $\S 3.1$, we write $N$ as

$$
\stackrel{\partial}{\longrightarrow} N_{00} \oplus N_{11} \stackrel{\partial}{\longrightarrow} N_{01} \oplus N_{10} \stackrel{\partial}{\longrightarrow} \text {. }
$$

Note that ${ }_{a} P \otimes_{A} P_{a} \cong \mathbb{C}(a) \oplus \mathbb{C} X_{a}$ is a two-dimensional vector space. Let $\zeta: N_{11} \rightarrow N_{00}$ be the map

$$
N_{11} \cong A \stackrel{-\Delta_{a}}{\longrightarrow} P_{a} \otimes{ }_{a} P \longrightarrow P_{a} \otimes{ }_{a} P \otimes_{A} P_{a} \otimes{ }_{a} P,
$$

where the last map takes $u_{1} \otimes u_{2}$ to $u_{1} \otimes(a) \otimes u_{2}$. Let $N^{\prime}=\left\{u+\zeta(u) \mid u \in N_{11}\right\}$. It is a subbimodule of $N_{11}$ isomorphic to $A$, and $\partial N^{\prime}=0$.

Let $N^{\prime \prime}=P_{a} \otimes(a) \otimes{ }_{a} P$. It is a subbimodule of $N_{00}$. 


\section{Frenkel, M. Khovanov and O. Schiffmann}

Lemma 6.2. $N$ is a direct sum of its three subduplexes

$$
\begin{gathered}
T_{-1}=\left\{\longrightarrow N_{10} \longrightarrow \partial N_{10} \longrightarrow\right\} \\
T_{0}=\left\{\longrightarrow N^{\prime} \longrightarrow 0 \longrightarrow\right\}, \\
T_{1}=\left\{\longrightarrow N^{\prime \prime} \longrightarrow \partial N^{\prime \prime} \longrightarrow\right\}
\end{gathered}
$$

Since $\partial$ is injective on $N_{10}$ and on $N^{\prime \prime}$, and both $N_{10}$ and $N^{\prime \prime}$ are projective bimodules, the duplexes $T_{-1}$ and $T_{1}$ are stably equivalent to the zero duplex. Therefore, $N$ is equivalent in the stable category to the bimodule duplex $\{\longrightarrow A \longrightarrow 0 \longrightarrow\}$.

Remarks. (i) Proposition 6.1 says that the functor $\mathcal{R}_{a}^{2}$

$$
\mathcal{K}_{2}(A, c) \stackrel{\mathcal{R}_{a}}{\longrightarrow} \mathcal{K}_{2}\left(A, s_{a}(c)\right) \stackrel{\mathcal{R}_{a}}{\longrightarrow} \mathcal{K}_{2}(A, c)
$$

is isomorphic to the identity functor, as long as $s_{a}(c) \neq c$ (equivalently, if $c_{a} \neq 0$ ).

(ii) The isomorphism (6.5) holds for $x=0$ as well, if we use (6.1), with $x=0$, to define one of the duplexes on the left-hand side of (6.5) and (6.2) to define the other.

Proposition 6.2. If $a$ and $b$ are not connected by an edge, for any $x, y \in \mathbb{C}$ there is an isomorphism of bimodule duplexes

$$
C_{a, x} \otimes_{A} C_{b, y} \cong C_{b, y} \otimes_{A} C_{a, x} .
$$

Proof. Since ${ }_{a} P \otimes_{A} P_{b} \cong 0 \cong{ }_{b} P \otimes_{A} P_{a}$, left- and right-hand sides of (6.7) are isomorphic to

$$
\stackrel{m_{a}+m_{b}}{\longrightarrow} A \stackrel{x \Delta_{a}+y \Delta_{b}}{\longrightarrow}\left(P_{a} \otimes{ }_{a} P\right) \oplus\left(P_{b} \otimes{ }_{b} P\right) \stackrel{m_{a}+m_{b}}{\longrightarrow} \text {. }
$$

Proposition 6.3. If $a$ and $b$ are connected by one edge, for any $x, y \in \mathbb{C}$ there is an isomorphism in the stable category of bimodule duplexes

$$
C_{a, y} \otimes_{A} C_{b, x+y} \otimes_{A} C_{a, x} \cong C_{b, x} \otimes_{A} C_{a, x+y} \otimes_{A} C_{b, y} .
$$

Proof. Denote by $N$ the duplex on the left-hand side of (6.8). It is built out of eight bimodules

$$
N_{i j k}=C_{a, y}^{i} \otimes_{A} C_{b, x+y}^{j} \otimes_{A} C_{a, x}^{k}, \quad i, j, k \in\{0,1\} .
$$

The differential $\partial$ of $N$ is injective on $N_{000}$ (the component $N_{000} \rightarrow N_{010}$ of $\partial$ is already injective). Let

$$
T_{-1}=\left\{\stackrel{\partial}{\longrightarrow} N_{000} \stackrel{\partial}{\longrightarrow} \partial N_{000} \stackrel{\partial}{\longrightarrow}\right\}
$$

be the subduplex of $N$ generated by $N_{000}$.

Let $N^{\prime}=P_{a} \otimes(a) \otimes{ }_{a} P \subset P_{a} \otimes{ }_{a} P \otimes_{A} P_{a} \otimes{ }_{a} P \cong N_{010}$. The differential is injective on $N_{010}$ (since the component $N_{010} \rightarrow N_{110}$ of $\partial$ is injective). Let

$$
T_{1}=\left\{\stackrel{\partial}{\longrightarrow} \partial N^{\prime} \stackrel{\partial}{\longrightarrow} N^{\prime} \stackrel{\partial}{\longrightarrow}\right\}
$$

be the subduplex of $N$ generated by $N_{010}$.

The algebra $A$ is Frobenius, and each projective $A$-module is injective. In particular, $P_{a}, P_{b}$ are injective $A$-modules, and $N^{\prime}, N_{000}$ (both isomorphic to $P_{a} \otimes{ }_{a} P$ ) are injective $A \otimes A^{o}$-modules (that is, injective $A$-bimodules). Moreover, $T_{1} \cap T_{-1}=0$. Applying Proposition 4.1 twice, we see that duplexes $N$ and $\widetilde{N}=N /\left(T_{-1} \oplus T_{1}\right)$ are isomorphic in the stable category of duplexes of bimodules.

Let $h$ be the edge with $o(h)=a$ and $i(h)=b$. The duplex $\widetilde{N}$ is isomorphic to

$$
\stackrel{\widetilde{\partial}^{1}}{\longrightarrow}\left(\begin{array}{c}
P_{a} \otimes{ }_{a} P \\
\oplus \\
P_{b} \otimes{ }_{b} P
\end{array}\right) \stackrel{\widetilde{\partial}^{0}}{\longrightarrow}\left(\begin{array}{c}
P_{a} \otimes{ }_{b} P \\
\oplus \\
A \\
\oplus \\
P_{b} \otimes{ }_{a} P
\end{array}\right) \stackrel{\widetilde{\partial}^{1}}{\longrightarrow}
$$




\section{Homological REALIZATION OF NAKAJIMA VARIETIES AND WEYL GROUP ACTIONS}

with the differential $\widetilde{\partial}$ given by matrices of bimodule maps

$$
\begin{gathered}
\widetilde{\partial}^{0}=\left(\begin{array}{cc}
\epsilon(\bar{h}) y \mathrm{id} \otimes \bar{h} & \epsilon(h) y \bar{h} \otimes \mathrm{id} \\
m_{a} & -m_{b} \\
\epsilon(h) x h \otimes \mathrm{id} & \epsilon(\bar{h}) x \mathrm{id} \otimes h
\end{array}\right) \\
\widetilde{\partial}^{1}=\left(\begin{array}{ccc}
\mathrm{id} \otimes h & (x+y) \Delta_{a} & -\bar{h} \otimes \mathrm{id} \\
h \otimes \mathrm{id} & -(x+y) \Delta_{b} & -\mathrm{id} \otimes \bar{h}
\end{array}\right) .
\end{gathered}
$$

The following example explains our notations: the entry $\epsilon(\bar{h}) y$ id $\otimes \bar{h}$ in the top left corner of (6.10) is a bimodule map $P_{a} \otimes{ }_{a} P \longrightarrow P_{a} \otimes_{b} P$ which takes $u \otimes v \in P_{a} \otimes{ }_{a} P$ to $\epsilon(\bar{h}) y u \otimes \bar{h} v \in P_{a} \otimes_{b} P$.

Denote by $M$ be the duplex on the right-hand side of (6.8). Since the right-hand side is obtained from the left-hand side by switching $a$ with $b, x$ with $y$, and $h$ with $\bar{h}$ we see that $M$ is isomorphic in the stable category to the duplex $\widetilde{M}$ defined by making these switchings in formulas (6.9), (6.10), (6.11). It is easy to check that duplexes $\widetilde{N}$ and $\widetilde{M}$ are isomorphic. Therefore, duplexes $N$ and $M$ are isomorphic in the stable category of duplexes.

We may restate the above results in the following form.

Theorem 1. The functors $\mathcal{R}_{a}: \underline{\operatorname{Mod}}_{2}\left(\widetilde{A}_{c}\right) \rightarrow \underline{\operatorname{Mod}}_{2}\left(\widetilde{A}_{s_{a}(c)}\right)$ define a braid group action on the family of categories $\underline{\mathbf{M o d}}_{2}\left(\widetilde{A}_{w(c)}\right)$ for $w \in \mathbf{W}$; in other words, we have, for any $c \in Z_{2}(A)$ isomorphisms of functors

$$
\mathcal{R}_{a} \circ \mathcal{R}_{b} \circ \mathcal{R}_{a} \simeq \mathcal{R}_{b} \circ \mathcal{R}_{a} \circ \mathcal{R}_{b}: \underline{\operatorname{Mod}}_{2}\left(\widetilde{A}_{c}\right) \rightarrow \underline{\operatorname{Mod}}_{2}\left(\widetilde{A}_{s_{a} s_{b} s_{a}(c)}\right)
$$

if $a$ and $b$ are connected by one edge, and

$$
\mathcal{R}_{a} \circ \mathcal{R}_{b} \simeq \mathcal{R}_{b} \circ \mathcal{R}_{a}: \underline{\operatorname{Mod}}_{2}\left(\widetilde{A}_{c}\right) \rightarrow \underline{\operatorname{Mod}}_{2}\left(\widetilde{A}_{s_{a} s_{b}(c)}\right)
$$

if $a$ and $b$ are not connected. Moreover, if $c$ lies in a generic orbit then this action factors through a Weyl group action, i.e. we have

$$
\mathcal{R}_{a} \circ \mathcal{R}_{a} \simeq \operatorname{Id}: \underline{\operatorname{Mod}}_{2}\left(\widetilde{A}_{c}\right) \rightarrow \underline{\operatorname{Mod}}_{2}\left(\widetilde{A}_{c}\right)
$$

for any $a \in I$.

Passing to the homotopy categories $\mathcal{K}_{2}(A, c)$ we obtain the following.

Theorem 2. The functors $\mathcal{R}_{a}: \mathcal{K}_{2}(A, c) \rightarrow \mathcal{K}_{2}\left(A, s_{a}(c)\right)$ define a braid group action on the family of categories $\mathcal{K}_{2}(A, w(c))$ for $w \in \mathbf{W}$. This action factors through to a Weyl group action if $c$ lies in a generic orbit.

Remark. In order to ensure that the above braid group action on the set of categories $\underline{\mathbf{M o d}}_{2}\left(\widetilde{A}_{w(c)}\right)$ or $\mathcal{K}_{2}(A, w(c))$ factors through the Weyl group it is enough to impose the following weaker condition: no point of the orbit of $c$ under $W$ is fixed by any of the reflexions $s_{a}$ for loopless vertices $a$.

\section{Weyl group actions on Nakajima varieties}

7.1 Let us say that $\boldsymbol{\zeta}_{\mathbb{C}}=-\sum_{a} \zeta_{\mathbb{C}, a} X_{a} \in A$ is generic if $\zeta_{\mathbb{C}}$ is generic in the sense of $\S 1.1$. If $N$ is any $\left(A, \boldsymbol{\zeta}_{\mathbb{C}}\right)$-duplex with $\boldsymbol{\zeta}_{\mathbb{C}}$ generic and $a \in I$ we set $\mathcal{R}_{a}(N)=C_{a, \zeta_{\mathbb{C}, a}} \otimes N$.

It follows from the results in $\S 6$ that this defines an action of the Weyl group $\mathbf{W}$ on the set of objects of $\underline{\operatorname{Mod}}_{2}\left(\widetilde{A}_{c}\right)$ for all generic $c$.

So let us assume that $\zeta_{\mathbb{C}}$ is generic, and let us identify the points of $\mathcal{M}_{\zeta}(\mathbf{v}, \mathbf{w})$ with isomorphism classes of data $(\underline{M}, \psi)$ as in Proposition 5.3.

Theorem 3. The functor $\mathcal{R}_{a}$ induces a bijection of sets from $\mathcal{M}_{\zeta}(\mathbf{v}, \mathbf{w})$ to $\mathcal{M}_{s_{a}(\zeta)}\left(s_{a}(\mathbf{v}, \mathbf{w})\right)$, which coincides with the isomorphism $\kappa_{s_{a}}$. 


\section{Frenkel, M. Khovanov and O. Schiffmann}

Proof. Let us describe the action of $\mathcal{R}_{a}$ in more details. For notational convenience we will write $N^{\oplus V}$ for the tensor product $N \otimes_{\mathbb{C}} V$ when $N$ is an $\widetilde{A}_{c}$-module and $V$ a $\mathbb{C}$-vector space. We will also denote by $(M, d)$ the objects of $\underline{\operatorname{Mod}}_{2}\left(\widetilde{A}_{c}\right)$. If $(M, d) \in \underline{\operatorname{Mod}}_{2}\left(\widetilde{A}_{\zeta_{\mathbb{C}}}\right)$ then by definition $\mathcal{R}_{a}(M, d)=$ $\left(M^{\prime}, d^{\prime}\right) \in \underline{\operatorname{Mod}}_{2}\left(\widetilde{A}_{s_{a}} \underline{\zeta_{\mathbb{C}}}\right)$ where

$$
M^{\prime}=\left(\begin{array}{c}
A \otimes M \\
\oplus \\
\left(P_{a} \otimes{ }_{a} P\right)[1] \otimes_{A} M
\end{array}\right)
$$

and

$$
d^{\prime}=\left(\begin{array}{cc}
1 \otimes d & m_{a} \otimes 1 \\
\Delta_{a} \otimes 1 & 1 \otimes d
\end{array}\right)
$$

Let us write

$$
M=\bigoplus_{k \in I}\left(P_{k}^{\oplus V_{k}} \oplus S_{k}^{\oplus W_{k}}[1]\right)
$$

Observe that

$$
A \otimes P_{a}^{\oplus V_{a}} \stackrel{\Delta_{a} \otimes 1}{\longrightarrow}\left(P_{a} \otimes{ }_{a} P\right)[1] \otimes_{A} P_{a}^{\oplus V_{a}}
$$

is injective. This implies that $A \otimes P_{a}^{\oplus V_{a}} \oplus d^{\prime}\left(A \otimes P_{a}^{\oplus V_{a}}\right)$ is stably trivial. Hence, by Proposition 4.1, $\left(M^{\prime}, d^{\prime}\right) \simeq\left(M^{\prime \prime}, d^{\prime \prime}\right)$ where $M^{\prime \prime}=M^{\prime} /\left(A \otimes P_{a}^{\oplus V_{a}} \oplus d^{\prime}\left(A \otimes P_{a}^{\oplus V_{a}}\right)\right)$. A direct computation shows that

$$
\left(P_{a} \otimes_{a} P\right)[1] \otimes_{A} M=\left(\begin{array}{c}
P_{a}^{\oplus V_{a}}[1] \\
\oplus \\
P_{a}^{\oplus V_{a}}[1] \\
\oplus \\
P_{a}^{\oplus W_{a}} \\
\oplus \\
\bigoplus P_{a}^{\oplus V_{b}}
\end{array}\right)
$$

and

We have

$$
M^{\prime \prime} \simeq\left(\begin{array}{c}
\bigoplus_{k \neq a} P_{k}^{\oplus V_{k}} \\
\oplus \\
\bigoplus_{k \neq a} S_{k}^{\oplus W_{k}} \\
\oplus \\
S_{a}^{\oplus W_{a}} \\
\oplus \\
P_{a}[1]^{\oplus V_{a}} \\
\oplus \\
P_{a}^{\oplus W_{a}} \\
\oplus \\
\bigoplus_{b-a}^{\oplus P_{a}^{\oplus V_{b}}}
\end{array}\right) .
$$

$$
d^{\prime \prime}=\left(\begin{array}{cccccc}
B & i & 0 & 0 & -B i & \lambda_{a} \mathrm{Id}-\epsilon B B \\
j & 0 & 0 & 0 & 0 & 0 \\
0 & 0 & 0 & 0 & \lambda_{a} \mathrm{Id}-j i & -j B \\
0 & 0 & 0 & 0 & -i & 0 \\
0 & 0 & \mathrm{Id} & j & 0 & 0 \\
\mathrm{Id} & 0 & 0 & B & 0 & 0
\end{array}\right)
$$




\section{Homological ReAlization of NAKAJIMA VARIETIES AND WEYL Group ACTIONS}

From the relation $\mu(B, i, j)=\lambda$ and from the fact that $\lambda$ is generic it follows that the fourth column of (7.2) is nonsingular. In particular, $d_{\mid P_{a}[1] \oplus V_{a}}^{\prime \prime}$ is injective and $P_{a}[1]^{\oplus V_{a}} \oplus d^{\prime \prime}\left(P_{a}[1]^{\oplus V_{a}}\right)$ is stably trivial.

Thus, $\left(M^{\prime \prime}, d^{\prime \prime}\right)$ is isomorphic, in $\underline{\operatorname{Mod}}_{2}\left(\widetilde{A}_{s_{a}(\underline{\mathbb{C}})}\right)$, to

$$
\left(M^{\prime \prime \prime}, d^{\prime \prime \prime}\right)=\left(M^{\prime \prime} /\left(P_{a}[1]^{\oplus V_{a}} \oplus d^{\prime \prime}\left(P_{a}[1]^{\oplus V_{a}}\right), d^{\prime \prime}\right)\right) .
$$

Moreover, there is a canonical isomorphism $u: R((M, d)) \simeq R\left(\left(M^{\prime}, d^{\prime}\right)\right) \simeq R\left(\left(M^{\prime \prime \prime}, d^{\prime \prime \prime}\right)\right)$ and we may set $\psi^{\prime}=u \circ \psi$. Let

$$
\left(B^{\prime}, i^{\prime}, j^{\prime}\right) \in \mathcal{M}_{s_{a}(\zeta)}\left(s_{a}(\mathbf{v}, \mathbf{w})\right)
$$

be the element corresponding to $\left(M^{\prime \prime \prime}, d^{\prime \prime \prime}, \psi^{\prime}\right)$. Comparing with the construction of $\S 1.4$ we see that $\kappa_{s_{a}}(B, i, j)=\left(B^{\prime}, i^{\prime}, j^{\prime}\right)$, which proves the theorem.

\section{Nakajima varieties for affine quivers}

In this section we restrict ourselves to the case when $(I, E)$ is an affine bipartite Dynkin diagram, and reinterpret the above construction in terms of the McKay correspondence. This section, and the following section, can be read independently of the rest of the paper, with the exception of $\S 9.3$.

8.1 Let $\{ \pm 1\} \subset \Gamma \subset S L(2, \mathbb{C})$ be a finite group and let $\left\{\rho_{a}\right\}_{a \in I}$ be the set of its irreducible representations. We also let $\rho_{0}$ and $\rho$ be the trivial (respectively the natural two-dimensional) representation. Let $\mathbf{Q}=(I, E)$ be the (unoriented) affine quiver associated to $\Gamma$ via the McKay correspondence, with $I$ as the set of vertices and with $T_{a b}$ arrows between $a$ and $b$, where

$$
T_{a b}=\operatorname{dim} \operatorname{Hom}_{\Gamma}\left(\rho_{a} \otimes \rho, \rho_{b}\right) .
$$

8.2 Let us consider the algebra $A_{\Gamma}:=\Lambda \mathbb{C}^{2} \rtimes \mathbb{C}[\Gamma]$, and set $\widetilde{A}_{\Gamma}=A_{\Gamma} \dot{\otimes} \mathbb{C}[d] / d^{2} \simeq \Lambda \mathbb{C}^{3} \rtimes \Gamma$ with relations $d z=-z d$ for any $z \in \mathbb{C}^{2}$ and $d \gamma=\gamma d$ for any $\gamma \in \Gamma$. Both $A_{\Gamma}$ and $\widetilde{A}_{\Gamma}$ are naturally $\mathbb{Z}$-graded. We denote by $\operatorname{Mod}\left(A_{\Gamma}\right)$ and $\operatorname{Mod}_{2}\left(A_{\Gamma}\right)\left(\right.$ respectively $\operatorname{Mod}\left(\widetilde{A}_{\Gamma}\right)$ and $\left.\operatorname{Mod}_{2}\left(A_{\Gamma}\right)\right)$ the categories of $\mathbb{Z}$-graded and $\mathbb{Z} / 2 \mathbb{Z}$-graded $A_{\Gamma}$-modules (respectively $\widetilde{A}_{\Gamma}$-modules). We will reformulate the definition of $\mathcal{M}_{\zeta}(\mathbf{v}, \mathbf{w})$ entirely in terms of representation theory of $A_{\Gamma}$ and $\widetilde{A}_{\Gamma}$.

The link with the setting of $\S 5$ is as follows. Let $I^{ \pm}$be the set of indices $a$ such that $\rho_{a}(-1)= \pm 1$. Then $I=I^{+} \sqcup I^{-}$and

$$
T_{a b} \neq 0 \Rightarrow a \in I^{+}, b \in I^{-} \quad \text { or } \quad a \in I^{-}, b \in I^{+} .
$$

In particular, the Dynkin diagram $(I, E)$ is bipartite. Write $A(\mathbf{Q})$ for the zigzag algebra corresponding to $(I, E)$. Recall that $\left\{\rho_{a}\right\}_{a \in I}$ denotes the set of simple left $\Gamma$-modules. Then $\left\{\rho_{a}^{*}\right\}$ is the set of right simple $\Gamma$-modules. Consider the right projective $A_{\Gamma}$-modules ${ }_{a} \mathbf{P}=\rho_{a}^{*} \otimes \Lambda \mathbb{C}^{2}$, and put $\mathbf{P}=\bigoplus_{a}{ }_{a} \mathbf{P}$.

It is easy to check that $A(\Gamma) \simeq \operatorname{End}_{A_{\Gamma}}(\mathbf{P})$. Moreover, the functor $\mathbf{P} \otimes-$ induces a Morita equivalence

$$
\operatorname{Mod}\left(A_{\Gamma}\right) \simeq \operatorname{Mod}(A(\mathbf{Q}))
$$

8.3 Note that $A_{\Gamma}$ and $\widetilde{A}_{\Gamma}$ are symmetric algebras. In particular, $A_{\Gamma}$ and $\widetilde{A}_{\Gamma}$ are self-injective algebras (i.e projective and injective objects coincide). If $M$ is a graded $A_{\Gamma}$ (respectively $\widetilde{A}_{\Gamma}$ )-module then the graded dual space $M^{*}$ is again an $A_{\Gamma}$ (respectively $\widetilde{A}_{\Gamma}$ )-module.

If $U$ is any $\Gamma$-module, we will regard $\Lambda \mathbb{C}^{2} \otimes U$ and $\left(\Lambda \mathbb{C}^{2} \dot{\otimes} \mathbb{C}[d] / d^{2}\right) \otimes U$ as graded $A_{\Gamma}$-module and $\widetilde{A}_{\Gamma}$-module, respectively, where $\Lambda^{0} \mathbb{C}^{2} \otimes U$ is placed in degree 0 . Note that any projective 


\section{Frenkel, M. Khovanov and O. Schiffmann}

indecomposable $A_{\Gamma}$-module (respectively $\widetilde{A}_{\Gamma}$-module) is isomorphic to $\left(\Lambda \mathbb{C}^{2} \otimes \rho_{a}\right)[n]$ (respectively $\left.\left(\left(\Lambda \mathbb{C}^{2} \dot{\otimes} \mathbb{C}[d] / d^{2}\right) \otimes \rho_{a}\right)[n]\right)$ for some $a \in I$ and $n \in \mathbb{Z}$.

Let us fix a basis $\{x, y\}$ in $\mathbb{C}^{2}$. For any $a \in I$, let us fix intertwiners

$$
\bigoplus_{(a, b) \in H} \varphi_{a b}: \bigoplus_{(a, b) \in H} \rho_{b} \stackrel{\sim}{\rightarrow} \mathbb{C}^{2} \otimes \rho_{a}
$$

Define a function $\epsilon: H \rightarrow \mathbb{C}^{*}$ by the following condition: $\pi \circ \varphi_{b a} \circ \varphi_{a b}=\epsilon_{(a, b)} x \wedge y$, where $\pi$ : $\mathbb{C}^{2} \otimes \mathbb{C}^{2} \otimes \rho_{b} \rightarrow \Lambda^{2} \mathbb{C}^{2} \otimes \rho_{b}$ is the projection. Note that $\epsilon(h)+\epsilon(\bar{h})=0$ for any $h \in H$.

8.4 Let $\underline{\operatorname{Mod}}\left(A_{\Gamma}\right)$ denote the stable category of $\mathbb{Z}$-graded $A_{\Gamma}$-modules (see $\S 4$.).

Lemma 8.1. Let $U$ be a $\Gamma$-module and let us consider it as a $A_{\Gamma}$ module by trivially extending the action to $A_{\Gamma}$. If $M \simeq U$ in $\underline{\operatorname{Mod}}\left(A_{\Gamma}\right)$ then $M \simeq U \oplus P$ in $\operatorname{Mod}\left(A_{\Gamma}\right)$ for some projective module $P$.

Proof. Let $f: M \rightarrow U$ and $f^{\prime}: U \rightarrow M$ such that $f f^{\prime}=\operatorname{Id}_{U}$ and $f^{\prime} f=\operatorname{Id}_{M}$ in $\underline{\operatorname{Mod}}\left(A_{\Gamma}\right)$. Note that, for any projective module $P$, any composition of morphisms $U \rightarrow P \rightarrow U$ is zero. Hence, $\operatorname{Hom}_{\underline{\operatorname{Mod}}\left(A_{\Gamma}\right)}(U, U)=\operatorname{Hom}_{A_{\Gamma}}(U, U)$ and $f f^{\prime}=\operatorname{Id}_{U}$ in $\operatorname{Mod}\left(A_{\Gamma}\right)$. However, then $M \simeq U \oplus \operatorname{Ker} f$ in $\operatorname{Mod}\left(A_{\Gamma}\right)$ and the lemma follows.

Similarly, we let $\underline{\operatorname{Mod}}\left(\widetilde{A}_{\Gamma}\right)$ stand for the stable category of $\mathbb{Z}$-graded $\widetilde{A}_{\Gamma}$-modules. Replacing $\mathbb{Z}$ by $\mathbb{Z} / 2 \mathbb{Z}$, we also define the categories $\underline{\operatorname{Mod}}_{2}\left(A_{\Gamma}\right)$ and $\underline{\operatorname{Mod}}_{2}\left(\widetilde{A}_{\Gamma}\right)$. The stable categories $\underline{\operatorname{Mod}}\left(A_{\Gamma}\right)$ and $\underline{\operatorname{Mod}}\left(\widetilde{A}_{\Gamma}\right)$ are endowed with structures of triangulated categories (see [Hap88]).

Note that $\widetilde{A}_{\Gamma}$ is a free $A_{\Gamma}$-module. This gives rise to functors

$$
R: \underline{\operatorname{Mod}}\left(\widetilde{A}_{\Gamma}\right) \rightarrow \underline{\operatorname{Mod}}\left(A_{\Gamma}\right), \quad R: \underline{\operatorname{Mod}}_{2}\left(\widetilde{A}_{\Gamma}\right) \rightarrow \underline{\operatorname{Mod}}_{2}\left(A_{\Gamma}\right) .
$$

8.5 In this section we give the realization of $\mathcal{M}_{\zeta}(\mathbf{v}, \mathbf{w})$ in the $\S 1.1$ case (ii), i.e. $\zeta_{\mathbb{R}}$ is arbitrary and $\zeta_{\mathbb{C}}$ is generic.

For every $a \in I$ we let $p_{a} \in \mathbb{C}[\Gamma]$ be the (central) primitive idempotent corresponding to $\rho_{a}$. Set $c_{a}=x \wedge y \cdot \underline{p}_{a}$. Then $\{1\} \cup\left\{c_{a}\right\}_{a \in I}$ forms a basis of the center of $A_{\Gamma}$. Consider the following deformation of $\widetilde{A}_{\Gamma}$ :

$$
\widetilde{A}_{\Gamma, \zeta_{\mathbb{C}}}=\left(\Lambda \mathbb{C}^{2} \rtimes \Gamma\right) \dot{\otimes} \mathbb{C}[d] /\left\langle d^{2}-\sum_{a} \zeta_{\mathbb{C}, a} c_{a}\right\rangle
$$

Let $\underline{\operatorname{Mod}}_{2}\left(\widetilde{A}_{\Gamma, \zeta \mathbb{C}}\right)$ be the stable categories of $\mathbb{Z} / 2 \mathbb{Z}$-graded $\widetilde{A}_{\Gamma, \zeta_{\mathbb{C}}}$-modules. As in $\S 8.4$, the embedding $A_{\Gamma} \subset \widetilde{A}_{\Gamma, \zeta_{\mathbb{C}}}$ gives rise to a restriction functor $R: \underline{\operatorname{Mod}}_{2}\left(\widetilde{A}_{\Gamma, \zeta_{\mathbb{C}}}\right) \rightarrow \underline{\operatorname{Mod}}_{2}\left(A_{\Gamma}\right)$. As in the undeformed case, the algebra $\widetilde{A}_{\Gamma, \zeta_{\mathbb{C}}}$ is symmetric and self-injective.

Let us fix w $\in \mathbb{N}^{I}, W=\bigoplus_{a} W_{a}$ such that $\operatorname{dim} W=\mathbf{w}$ and let $\mathbb{W}=\bigoplus_{a} W_{a} \otimes \rho_{a}$ be the corresponding $\Gamma$-module. We will regard $\mathbb{W}$ as a graded $A_{\Gamma}$-module, where $\Lambda \mathbb{C}^{2}$ acts trivially, placed in degree 0 . Let $\mathcal{N}_{\zeta}(\mathbf{w})$ be the set of pairs $(u, M)$ where $M \in \underline{\operatorname{Mod}}_{2}\left(\widetilde{A}_{\Gamma, \zeta_{\mathbb{C}}}\right)$ and $u: \mathbb{W} \rightarrow R(M)$ is an isomorphism.

Lemma 8.2. Let $M$ be any $\widetilde{A}_{\Gamma, \zeta_{\mathbb{C}}}$-module such that $R(M) \simeq 0$. Then $M$ is projective.

Proof. By Lemma 8.1 we have $M \simeq\left(\Lambda \mathbb{C}^{2} \otimes \mathbb{V}_{1}\right) \oplus\left(\Lambda \mathbb{C}^{2} \otimes \mathbb{V}_{2}\right)[-1]$ for some $\Gamma$-modules $\mathbb{V}_{1}$ and $\mathbb{V}_{2}$. We may assume that

$$
d\left(\mathbb{V}_{1}\right) \subset \mathbb{C}^{2} \otimes \mathbb{V}_{1}
$$

Indeed, any $v \in \mathbb{V}_{1}$ not satisfying (8.4) generates a projective submodule $M^{\prime}$ of $M$. Consider the linear map $s: \Lambda^{2} \mathbb{C}^{2} \rightarrow \mathbb{C}, x \wedge y \mapsto 1$. From (8.4) and the relation $d z=-z d$ for $z \in \mathbb{C}^{2}$ we deduce 


\section{Homological REALIZATION OF NAKAJIMA VARIETIES AND WEYL GROUP ACTIONS}

that $\operatorname{Tr}_{\mid V}\left(s \circ d^{2}\right)=0$. However, by definition,

$$
\operatorname{Tr}_{\mid V}\left(s \circ d^{2}\right)=\sum_{a} \zeta_{\mathbb{C}, a} \operatorname{dim} \operatorname{Hom}\left(\rho_{a}, \mathbb{V}_{1}\right),
$$

and the genericity of $\zeta_{\mathbb{C}}$ implies that $\mathbb{V}_{1}=0$. Similarly, $\mathbb{V}_{2}=0$ and we are done.

THEOREM 4. There is a natural bijection between the set of isomorphism classes of elements in $\mathcal{N}_{\zeta}(\mathbf{w})$ and the set of points of $\bigsqcup_{\mathbf{v}} \mathcal{M}_{\zeta}(\mathbf{v}, \mathbf{w})$.

Proof. Let $(u, M) \in \mathcal{N}_{\zeta}(\mathbf{w})$. By Lemma 8.1, we may assume that $M=\left(\Lambda \mathbb{C}^{2} \otimes \mathbb{V}_{1}[1]\right) \oplus \mathbb{W} \oplus\left(\Lambda \mathbb{C}^{2} \otimes \mathbb{V}_{0}\right)$

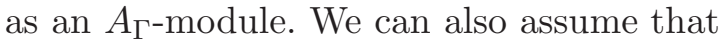

$$
d\left(\mathbb{V}_{0}\right) \subset\left(\mathbb{C}^{2} \otimes \mathbb{V}_{0}\right) \oplus\left(\Lambda^{2} \mathbb{C}^{2} \otimes \mathbb{V}_{1}\right) .
$$

Indeed if not then any element $v_{0} \in \mathbb{V}_{0}$ not satisfying (8.5) will generate a projective $\widetilde{A}_{\Gamma, \zeta_{\mathbb{C}}}$-module $N_{1}$ and $M \simeq M / N_{1}$ in $\underline{\operatorname{Mod}}_{2}\left(\widetilde{A}_{\Gamma, \zeta \mathbb{C}}\right)$. Similarly, we can assume that

$$
d\left(\mathbb{V}_{1}\right) \subset\left(\mathbb{C}^{2} \otimes \mathbb{V}_{1}\right) \oplus\left(\Lambda^{2} \mathbb{C}^{2} \otimes \mathbb{V}_{0}\right) \oplus \mathbb{W} .
$$

However, then $d\left(\Lambda^{2} \mathbb{C}^{2} \otimes \mathbb{V}_{1}\right)=0$ and, in particular, the composition of maps $\mathbb{V}_{1} \stackrel{d}{\rightarrow} \mathbb{C}^{2} \otimes \mathbb{V}_{1} \stackrel{d}{\rightarrow}$ $\Lambda^{2} \mathbb{C}^{2} \otimes \mathbb{V}_{1}$ endows $\Lambda \mathbb{C}^{2} \otimes \mathbb{V}_{1}$ with a structure of $\widetilde{A}_{\Gamma, \zeta_{\mathbb{C}}}$-module. By Lemma 8.2, this implies that $\mathbb{V}_{1}=0$.

Let us fix some decomposition

$$
\mathbb{V}=\bigoplus_{a} V_{a} \otimes \rho_{a}
$$

and set $V=\bigoplus_{a} V_{a}$. Let us split the map $d: \mathbb{V} \rightarrow \mathbb{C}^{2} \otimes \mathbb{V} \oplus \mathbb{W}$ as $d=d_{0}+d_{1}$ where $d_{0}: \mathbb{V} \rightarrow \mathbb{C}^{2} \otimes \mathbb{V}$ and $d_{1}: \mathbb{V} \rightarrow \mathbb{W}$. Then the maps $d_{0}$ and $d_{1}$ give rise, via the identification (8.7), the fixed intertwiners (8.3) and the map $u$, to elements $B=\bigoplus_{h \in H} x_{h} \in E(V, V)$ and $j \in L(V, W)$, respectively. Similarly, the map $d: \mathbb{C}^{2} \otimes \mathbb{V} \oplus \mathbb{W} \rightarrow \Lambda^{2} \mathbb{C}^{2} \otimes \mathbb{V} \simeq \mathbb{V}$ gives rise to elements $C=\bigoplus_{h \in H} y_{h} \in E(V, V)$ and $i \in L(W, V)$. From the relation $d z=-z d$ for $u \in \mathbb{C}^{2}$ we deduce that $y_{h}=\epsilon(h) x_{h}$ where $\epsilon: H \rightarrow \mathbb{C}^{*}$ is defined in $\S$ 8.3. Similarly, from $d^{2}=\sum_{a} \zeta_{\mathbb{C}, a} c_{a}$ we deduce the relation $\mu(B, i, j)=\boldsymbol{\zeta}_{\mathbb{C}}$.

Note that the assignment $M \rightarrow(B, i, j)$ depends on a choice of the decomposition (8.7), but that two such decompositions give rise to the same element in $\mathcal{M}_{\zeta}(\mathbf{v}, \mathbf{w})$. Hence we have obtained in this way a well-defined map from the set of isomorphism classes of objects in $\mathcal{N}_{\zeta}(\mathbf{w})$ to $\bigsqcup_{\mathbf{v}} \mathcal{M}_{\zeta}(\mathbf{v}, \mathbf{w})$. Conversely, it is clear that any point $(B, i, j) \in \mathcal{M}_{\zeta}(\mathbf{v}, \mathbf{w})$ gives rise, via the above construction, to an $\widetilde{A}_{\Gamma, \zeta_{\mathbb{C}}}$-module structure on the $A_{\Gamma}$-module $M=\Lambda\left(\mathbb{C}^{2} \otimes \mathbb{V}\right)[1] \oplus \mathbb{W}$. Moreover, this module $M$ is equipped with a canonical isomorphism $u: \mathbb{W} \stackrel{\sim}{\rightarrow} R(M)$. Thus, $M \in \mathcal{N}_{\zeta}(\mathbf{w})$. This map assigns distinct points in $\mathcal{M}_{\zeta}(\mathbf{v}, \mathbf{w})$ to nonisomorphic objects in $\mathcal{N}_{\zeta}(\mathbf{w})$ and the theorem follows.

Remark. Theorem 4 is equivalent to Proposition 5.3.

\section{Koszul duality and sheaves on $\mathbb{P}^{2}$}

9.1 In this section we clarify the relation between the construction of $\S 8$ and the moduli space of coherent sheaves on some noncommutative deformations of the projective plane, as studied in [BGK].

Let $\underline{\operatorname{Mod}}^{0}\left(\widetilde{A}_{\Gamma}\right)$ be the full subcategory of $\underline{\operatorname{Mod}}\left(\widetilde{A}_{\Gamma}\right)$ consisting of objects $V$ satisfying

$$
\operatorname{Ext}_{\widetilde{A}_{\Gamma}}^{l+n}(\mathbb{C}, V[-l]) \neq 0 \Rightarrow n=0,
$$

where $\mathbb{C}$ denotes the trivial module. The category $\underline{\operatorname{Mod}}^{0}\left(A_{\Gamma}\right)$ is defined in a similar way. 


\section{Frenkel, M. Khovanov and O. Schiffmann}

To any $\widetilde{A}_{\Gamma}$ module $V=\bigoplus_{n \in \mathbb{Z}} V_{n}$ is associated a complex of $\Gamma$-equivariant coherent sheaves on $\mathbb{P}^{2}$

$$
\cdots \stackrel{d}{\rightarrow} L_{i}\left(V^{*}\right) \stackrel{d}{\rightarrow} L_{i+1}\left(V^{*}\right) \stackrel{d}{\rightarrow} \cdots
$$

where $L_{i}\left(V^{*}\right)=\left(V^{*}\right)_{i} \otimes \mathcal{O}(i)$ and where the differential is defined by $(d \zeta)(x)=x \cdot \zeta(x)$ for $x \in \mathbb{P}^{2}$ and any section $\zeta \in \Gamma\left(L_{i}(V)\right)$. Here $V^{*}$ denotes the $\widetilde{A}_{\Gamma}$-module dual to $V$. A well-known theorem of Bernštern et al. [BGG78] generalizing the classical Koszul duality between $\mathbb{C}[x, y, z]$ and $\Lambda \mathbb{C}^{3}$ asserts that the assignment $\Phi: V \rightarrow L_{\bullet}\left(V^{*}\right)$ induces an equivalence between $\underline{\operatorname{Mod}}\left(\widetilde{A}_{\Gamma}\right)^{o p}$ and the $\Gamma$-equivariant derived category $D_{\Gamma}^{b}\left(\operatorname{Coh}\left(\mathbb{P}^{2}\right)\right)$ of coherent sheaves on $\mathbb{P}^{2}$. In particular, under this equivalence condition (9.1) corresponds to $H^{i}\left(L_{\bullet}\left(V^{*}\right)\right) \neq 0 \Rightarrow i=0$ (see [BGS96, § 2.13]), and $\underline{\operatorname{Mod}}^{0}\left(\widetilde{A}_{\Gamma}\right)^{o p}$ is equivalent to the category $\operatorname{Coh}_{\Gamma}\left(\mathbb{P}^{2}\right)$ of $\Gamma$-equivariant coherent sheaves on $\mathbb{P}^{2}$. Therefore, it is an abelian category. Similar statements hold for $\underline{\operatorname{Mod}}\left(A_{\Gamma}\right)$ and $D_{\Gamma}^{b}\left(\mathbb{P}^{1}\right)$.

The functor $R$ restricts to a functor $\underline{\operatorname{Mod}}^{0}\left(\widetilde{A}_{\Gamma}\right) \rightarrow \underline{\operatorname{Mod}}^{0}\left(A_{\Gamma}\right)$. It corresponds to the functor of restriction

$$
D_{\Gamma}^{b}\left(\operatorname{Coh}\left(\mathbb{P}^{2}\right)\right) \rightarrow D_{\Gamma}^{b}\left(\operatorname{Coh}\left(\mathbb{P}^{1}\right)\right)
$$

induced by the embedding $\mathbb{P}^{1} \simeq \mathbb{P}\left(\left(\mathbb{C}^{2}\right)^{*}\right) \hookrightarrow \mathbb{P}\left(\left(\mathbb{C}^{2} \oplus \mathbb{C} d\right)^{*}\right) \simeq \mathbb{P}^{2}$.

Let us denote by $\Pi_{\Gamma}$ the preprojective algebra of the affine quiver $(I, E)$ (see, e.g., [Maf02]). It is well known and easy to check that $A(\Gamma)$ is Koszul and that its quadratic dual is $\Pi_{\Gamma}$ (see, e.g., [HK01]). Thus, altogether we get the following diagram relating various algebras.

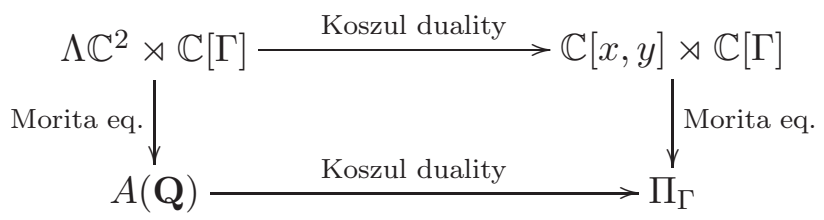

Similarly, there is the following diagram.

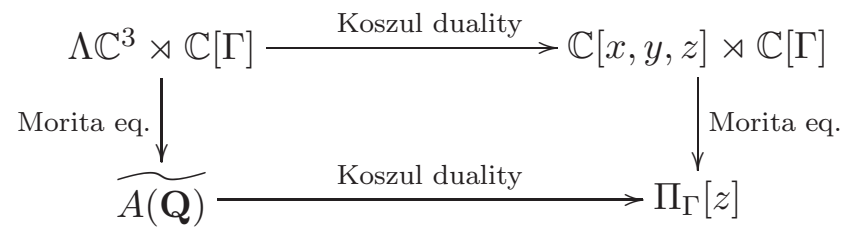

Our construction in $\S 8$ is based on a deformation of the left column of diagram (9.2). The corresponding right column consists of the homogeneous coordinate ring of the noncommutative $\mathbb{P}^{2}$ studied in [BGK], and the (graded) deformed preprojective algebra (see [CH98]).

9.2 In this section we show how to recover the description of Nakajima varieties as moduli space of torsion-free sheaves on $\mathbb{P}^{2}$ with fixed framing at $\infty$, using the representation theory of $\widetilde{A}_{\Gamma}$. This corresponds to $\S 1.1$ case (i), i.e. $\zeta_{\mathbb{C}}=0$ and $\zeta_{\mathbb{R}} \in\left(\mathbb{N}^{+}\right)^{I}$.

Let $\mathcal{T}$ be the full subcategory of $\underline{\operatorname{Mod}}^{0}\left(\widetilde{A}_{\Gamma}\right)$ consisting of modules $T$ such that $\Phi(T)$ is a torsion sheaf on $\mathbb{P}^{2}$. We will say that an object $M$ of $\underline{\operatorname{Mod}}_{\mathbb{Z}}^{0}\left(\widetilde{A}_{\Gamma}\right)$ is torsion-free if for any $T \in \mathcal{T}$ we have $\operatorname{Hom}_{\underline{\operatorname{Mod}}^{0}\left(\widetilde{A}_{\Gamma}\right)}(M, T)=0$.

Lemma 9.1. Let $N$ be a graded $\widetilde{A}_{\Gamma}$-module such that $N=\Lambda \mathbb{C}^{2} \otimes \mathbb{V}$ as a $A_{\Gamma}$-module. If $\mathbb{V} \neq\{0\}$ then $H^{0}(\Phi(N))$ is a nontrivial torsion sheaf.

Proof. This follows from a direct computation.

Lemma 9.2. Let $N \in \underline{\operatorname{Mod}}^{0}\left(\widetilde{A}_{\Gamma}\right)$ such that $N_{i}=0$ for $i>0$ and such that $R(N) \simeq 0$. Then $N \simeq 0$. 


\section{Homological ReAlization of NAKAJIMA VARIETIES AND WEYL Group ACTIONS}

Proof. Suppose $N \not 0$. By Lemma 8.1, $N$ decomposes as a $A_{\Gamma}$-module as $N=\bigoplus_{i=r}^{-2} \Lambda \mathbb{C}^{2} \otimes \mathbb{V}_{i}$ for some $\Gamma$-modules $\mathbb{V}_{i}$ and some $r \leqslant-2$. We can assume

$$
d\left(\mathbb{V}_{r}\right) \subset \mathbb{C}^{2} \otimes \mathbb{V}_{r} .
$$

Indeed, any $v_{r} \in \mathbb{V}_{r}$ not satisfying (a) generates a projective $\widetilde{A}_{\Gamma}$-module $N^{\prime}$ and $N \simeq N / N^{\prime}$ in $\underline{\operatorname{Mod}}^{0}\left(\widetilde{A}_{\Gamma}\right)$. However, then it follows from the previous lemma that $H^{-r}(\Phi(N)) \neq 0$, in contradiction with the assumption that $N \in \underline{\operatorname{Mod}}^{0}\left(\widetilde{A}_{\Gamma}\right)$.

As in $\S 8.4$ we fix $\mathbf{w} \in \mathbb{N}^{I}$ and associate to it a $\Gamma$-module $\mathbb{W}$. We will regard also $\mathbb{W}$ as a graded $A_{\Gamma}$-module, where $\Lambda \mathbb{C}^{2}$ acts trivially, placed in degree 0 . Note that $\mathbb{W}$ is naturally an object of $\underline{\operatorname{Mod}}^{0}\left(A_{\Gamma}\right)$. Let $\mathcal{N}(\mathbf{w})$ denote the set of pairs $(u, M)$ where $M \in \underline{\operatorname{Mod}}^{0}\left(\widetilde{A}_{\Gamma}\right)$ is torsion-free and $u: \mathbb{W} \stackrel{\sim}{\rightarrow} R(M)$ is an isomorphism. We say two elements $\left(u_{1}, M_{1}\right)$ and $\left(u_{2}, M_{2}\right)$ are isomorphic if there exists an isomorphism $j: M_{1} \rightarrow M_{2}$ such that $u_{2}=R(j) \circ u_{1}$.

TheOREM 5. There is a natural bijection between the set of isomorphism classes of elements in $\mathcal{N}(\mathbf{w})$ and the set of points of $\bigsqcup_{\mathbf{v}} \mathcal{M}_{\zeta}(\mathbf{v}, \mathbf{w})$.

Proof. Let $(u, M) \in \mathcal{N}(\mathbf{w})$. We first show the following.

Lemma 9.3. There exists a $\Gamma$-module $\mathbb{V}$ and $M^{\prime} \in \underline{\operatorname{Mod}}\left(\tilde{A}_{\Gamma}\right)$ such that $M \simeq M^{\prime}$ and $M^{\prime}=$ $\Lambda \mathbb{C}^{2} \otimes \mathbb{V}[+1] \oplus \mathbb{W}$ as an $A_{\Gamma-\text {-module. }}$

Proof. By Lemma 8.1, there exists $\Gamma$-modules $\mathbb{V}_{i}, i \in \mathbb{Z}$ such that $M \simeq \bigoplus_{i} \Lambda \mathbb{C}^{2} \otimes \mathbb{V}_{i}[-i] \oplus \mathbb{W}$ as an $A_{\Gamma}$-module. Observe that

$$
d\left(\mathbb{V}_{-1}\right) \subset \Lambda^{2} \mathbb{C}^{2} \otimes \mathbb{V}_{-2} \oplus \mathbb{C}^{2} \otimes \mathbb{V}_{-1} \oplus \mathbb{W} .
$$

Indeed, if not, then any $v_{-1} \in \mathbb{V}_{-1}$ such that (9.3) does not hold generates a projective submodule $N$ of $M$, and $M \simeq M / N$ in $\underline{\operatorname{Mod}}^{0}\left(\tilde{A}_{\Gamma}\right)$. Let $T$ be the $\widetilde{A}_{\Gamma}$-module obtained by restricting the

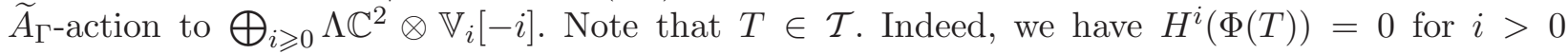
and $H^{i}(\Phi(T))=H^{i}(\Phi(M))=0$ for $i<0$, so that $T \in \underline{\operatorname{Mod}}^{0}\left(\widetilde{A}_{\Gamma}\right)$, and $H^{0}(\Phi(T))$ is torsion by Lemma 9.1. However, $M$ is assumed to be torsion-free. This forces $T \simeq 0$.

A reasoning similar to $(9.3)$ shows that

$$
d\left(\mathbb{V}_{-2}\right) \subset \mathbb{C}^{2} \otimes \mathbb{V}_{-2} \oplus \Lambda^{2} \mathbb{C}^{2} \otimes \mathbb{V}_{-3}
$$

Now observe that $N=\bigoplus_{i<-1} \Lambda \mathbb{C}^{2} \otimes \mathbb{V}_{i}$ is in $\underline{\operatorname{Mod}}^{0}\left(\widetilde{A}_{\Gamma}\right)$ and that $R(N) \simeq 0$. Thus $N \simeq 0$ by Lemma 9.2, and the lemma follows.

Note that by Lemma 9.2 again, the following holds:

$$
\text { for all } N \subset M, \quad R(N)=0 \Rightarrow N \simeq 0
$$

and that condition (9.4) is equivalent to the stability condition of $\S 1.1$, case (i).

The rest of the proof of the theorem now closely follows the proof of Theorem 4 .

Remark. The above description of torsion-free sheaves on $\mathbb{P}^{2}$ with fixed framing at infinity is equivalent to the classical one in terms of Beilinson's monads (see, e.g., [Nak99, Ch. 2]) but its derivation does not use spectral sequences.

From the above proof one easily deduces the following result.

Corollary 1. The variety $\mathcal{M}_{\zeta}(\mathbf{v}, \mathbf{w})$ is isomorphic to the set of all $\Lambda \mathbb{C}^{2} \rtimes \mathbb{C}[\Gamma]$-derivations (of degree one) of the module $\Lambda \mathbb{C}^{2} \otimes \mathbb{V} \oplus \mathbb{W}$ satisfying the following condition: if $\mathbb{V}^{\prime} \subset \mathbb{V}$ is a $\Gamma$-submodule such that $\Lambda \mathbb{C}^{2} \otimes \mathbb{V}^{\prime}$ is $d$-stable then $\mathbb{V}^{\prime}=0$. 


\section{Frenkel, M. Khovanov and O. Schiffmann}

9.3 Denote by $\iota_{a}: \operatorname{End}\left(\rho_{a}\right) \rightarrow \mathbb{C}[\Gamma] \simeq \bigoplus_{a} \operatorname{End}\left(\rho_{a}\right)$ the canonical embedding, and let $\pi_{a}: \mathbb{C}[\Gamma] \rightarrow$ $\operatorname{End}\left(\rho_{a}\right)$ be the canonical projection. We call

$$
m: \Lambda \mathbb{C}^{2} \otimes \mathbb{C}[\Gamma] \otimes \Lambda \mathbb{C}^{2} \rightarrow \Lambda \mathbb{C}^{2} \otimes \mathbb{C}[\Gamma]
$$

the multiplication map and we define

$$
\Delta: \Lambda \mathbb{C}^{2} \otimes \mathbb{C}[\Gamma] \rightarrow \Lambda \mathbb{C}^{2} \otimes \mathbb{C}[\Gamma] \otimes \Lambda \mathbb{C}^{2}
$$

to be its adjoint. Consider the following maps of $A_{\Gamma^{-}}$-bimodules:

$$
\begin{gathered}
d_{1}: \Lambda \mathbb{C}^{2} \otimes \rho_{a} \otimes \mathbb{C} \rho_{a}^{*} \otimes \Lambda \mathbb{C}^{2} \simeq \Lambda \mathbb{C}^{2} \otimes \operatorname{End}\left(\rho_{a}\right) \otimes \Lambda \mathbb{C}^{2} \stackrel{m \circ\left(1 \otimes \iota_{a} \otimes 1\right)}{\longrightarrow} \Lambda \mathbb{C}^{2} \otimes \mathbb{C}[\Gamma] \\
d_{2}: \Lambda \mathbb{C}^{2} \otimes \mathbb{C}[\Gamma] \stackrel{\left(1 \otimes \pi_{a} \otimes 1\right) \circ \Delta}{\longrightarrow} \Lambda \mathbb{C}^{2} \otimes \operatorname{End}\left(\rho_{a}\right) \otimes \Lambda \mathbb{C}^{2} .
\end{gathered}
$$

As in $\S 6$, this gives rise, for any $x \neq 0$ to a duplex of $A_{\Gamma \text {-bimodules }}$

$$
\mathbf{C}_{a, x}: \stackrel{d_{2}}{\rightarrow} \Lambda \mathbb{C}^{2} \otimes \operatorname{End}\left(\rho_{a}\right) \otimes \Lambda \mathbb{C}^{2} \stackrel{d_{1}}{\rightarrow} \Lambda \mathbb{C}^{2} \otimes \mathbb{C}[\Gamma] \stackrel{d_{2}}{\rightarrow} .
$$

One checks that the $A_{\Gamma}$ bimodule duplex $\mathbf{C}_{a, x}$ corresponds to the $A(\mathbf{Q})$-bimodule duplex $C_{a, x}$ under the equivalence $\operatorname{Mod}\left(A_{\Gamma}\right) \simeq \operatorname{Mod}(A(\mathbf{Q}))$. In particular, the collection of duplexes $\mathbf{C}_{a, x}$ satisfy the braid relations of $\S 6.3$ (in the stable category of bimodule duplexes). Thus, as in $\S 7$, tensoring by $\mathbf{C}_{a, x}$ for $a \in I$ and generic $x$ defines an action of the Weyl group $\mathbf{W}$ on the set of objects of $\underline{\operatorname{Mod}}_{2}\left(A_{\Gamma, \zeta_{\mathbb{C}}}\right)$ for all generic $\zeta_{\mathbb{C}}$.

In other words, (for generic $\zeta_{\mathbb{C}}$ ) and $a \in I$ we have a functor

$$
\mathcal{R}_{a}: \underline{\operatorname{Mod}}_{2}\left(\widetilde{A}_{\Gamma, \zeta_{\mathbb{C}}}\right) \rightarrow \underline{\operatorname{Mod}}_{2}\left(\widetilde{A}_{\Gamma, s_{a}(\zeta \mathbb{C}}\right),
$$

and the collection of such functors satisfy the braid relations. Moreover, there is a canonical natural transformation $R \circ \mathcal{R}_{a} \rightarrow R$, and for any given fixed $\mathbf{w} \in \mathbb{N}^{I}, \mathcal{R}_{a}$ acts on the set of objects of $\mathcal{N}_{\zeta}(\mathbf{w})$. The following proposition is a consequence of Theorem 1.

Proposition 9.1. The action of $\mathcal{R}_{a}$ on $\mathcal{N}_{\zeta}(\mathbf{w})$ coincides with the action of $\kappa_{a}$ under the identification $\mathcal{N}_{\zeta}(\mathbf{w}) \simeq \bigsqcup_{\mathbf{v}} \mathcal{M}_{\zeta}(\mathbf{v}, \mathbf{w})$.

\section{ACKNOWLEDGEMENTS}

Mikhail Khovanov would like to thank Igor Burban for interesting discussions about periodic triangulated categories. While working on this paper, Igor Frenkel and Mikhail Khovanov were partially supported by NSF grants DMS-0070551 and DMS-0104139.

\section{REFERENCES}

BGG78 I. N. Bernšteĭn, I. M. Gel'fand and S. I. Gel'fand, Algebraic vector bundles on $P^{n}$ and problems of linear algebra, Funktsional. Anal. i Prilozhen. 12 (1978), 66-67 (in Russian).

BGK V. Baranovsky, V. Ginzburg and A. Kuznetsov, Quiver varieties and a noncommutative $\mathbb{P}^{2}$, Compositio Math. 134 (2002), 283-318.

BGS96 A. Beilinson, V. Ginzburg and W. Soergel, Koszul duality patterns in representation theory, J. Amer. Math. Soc. 9 (1996), 473-527.

Bor88 R. Borcherds, Generalized Kac-Moody algebras, J. Algebra 115 (1988), 501-512.

CH98 W. Crawley-Boevey and M. Holland, Noncommutative deformations of Kleinian singularities, Duke Math. J. 92 (1998), 605-635.

Hap88 D. Happel, Triangulated categories in the representation theory of finite-dimensional algebras, London Mathematical Society Lecture Note Series, vol. 119 (Cambridge University Press, Cambridge, 1988). 


\section{Homological REALIZATION OF NAKAJIMA VARIETIES AND WEYL GROUP ACTIONS}

HK01 R. S. Huerfano and M. Khovanov, A category for the adjoint representation, J. Algebra 246 (2001), $514-542$.

KS02 M. Khovanov and P. Seidel, Quivers, Floer cohomology, and braid group actions, J. Amer. Math. Soc. 15 (2002), 203-271.

Kum02 S. Kumar, Kac-Moody groups, their flag varieties and representation theory (Birkhäuser, Boston, MA, 2002).

Lus00 G. Lusztig, Quiver varieties and Weyl group actions, Ann. Inst. Fourier (Grenoble) 50 (2000), 461-489.

Maf02 A. Maffei, A remark on quiver varieties and Weyl groups, Ann. Sc. Norm. Super. Pisa Cl. Sci. (5) 1 (2002), 649-686.

MS94 D. McDuff and D. Salamon, J-holomorphic curves and quantum cohomology, University Lecture Series, vol. 6 (American Mathematical Society, Providence, RI, 1994).

Nak94 H. Nakajima, Instantons on ALE spaces, quiver varieties, and Kac-Moody algebras, Duke Math. J. 76 (1994), 365-416.

Nak99 H. Nakajima, Lectures on Hilbert schemes of points on surfaces, University Lecture Series, vol. 18 (American Mathematical Society, Providence, RI, 1999).

Nak03 H. Nakajima, Reflection functors for quiver varieties and Weyl group actions, Math. Ann. 327 (2003), 671-721.

PX00 L. Peng and J. Xiao, Triangulated categories and Kac-Moody algebras, Invent. Math. 140 (2000), 563-603.

RZ03 R. Rouquier and A. Zimmermann, Picard groups for derived module categories, Proc. London Math. Soc. (3) 87 (2003), 197-225.

ST01 P. Seidel and R. Thomas, Braid group actions on derived categories of coherent sheaves, Duke Math. J. 108 (2001), 37-108.

Igor Frenkel

Department of Mathematics, Yale University, 10 Hillhouse Avenue, New Haven, CT 06520-8283, USA

Mikhail Khovanov mikhail@math.ucdavis.edu

Department of Mathematics, University of California, One Shields Avenue, Davis, CA 95616-8633, USA

Olivier Schiffmann schiffma@dma.ens.fr

Department of Mathematics, Yale University, 10 Hillhouse Avenue, New Haven, CT 06520-8283, USA and DMA Ens Paris, 45 rue d'Ulm, 75005 Paris, France 